\title{
Chemical Synthesis of the Antiviral Nucleotide Analogue ddhCTP
}

\author{
James. M. Wood ${ }^{1,2}$, Gary B. Evans ${ }^{1,2}$, Tyler L. Grove ${ }^{3}$, Steven C. Almo ${ }^{3}$, Scott A. \\ Cameron $^{1,2}$, Richard H. Furneaux ${ }^{1,2}$, Lawrence D. Harris ${ }^{1,2 *}$
}

${ }^{1}$ Ferrier Research Institute, Victoria University of Wellington, Wellington 6012, New Zealand ${ }^{2}$ The Maurice Wilkins Centre for Molecular Biodiscovery, The University of Auckland, Auckland, New Zealand ${ }^{3}$ Department of Biochemistry, Albert Einstein College of Medicine, Bronx, New York 10461, United States

Email: Lawrence.Harris@vuw.ac.nz

\section{Contents}

ORTEP Diagram, Crystal Data and Structure Refinement Table for Iodide 11 S2

${ }^{1} \mathrm{H},{ }^{13} \mathrm{C}\left\{{ }^{1} \mathrm{H}\right\}$ and ${ }^{31} \mathrm{P}\left\{{ }^{1} \mathrm{H}\right\}$ NMR Spectra of Novel Compounds S4 
ORTEP Diagram, Crystal Data and Structure Refinement Table for Iodide 11

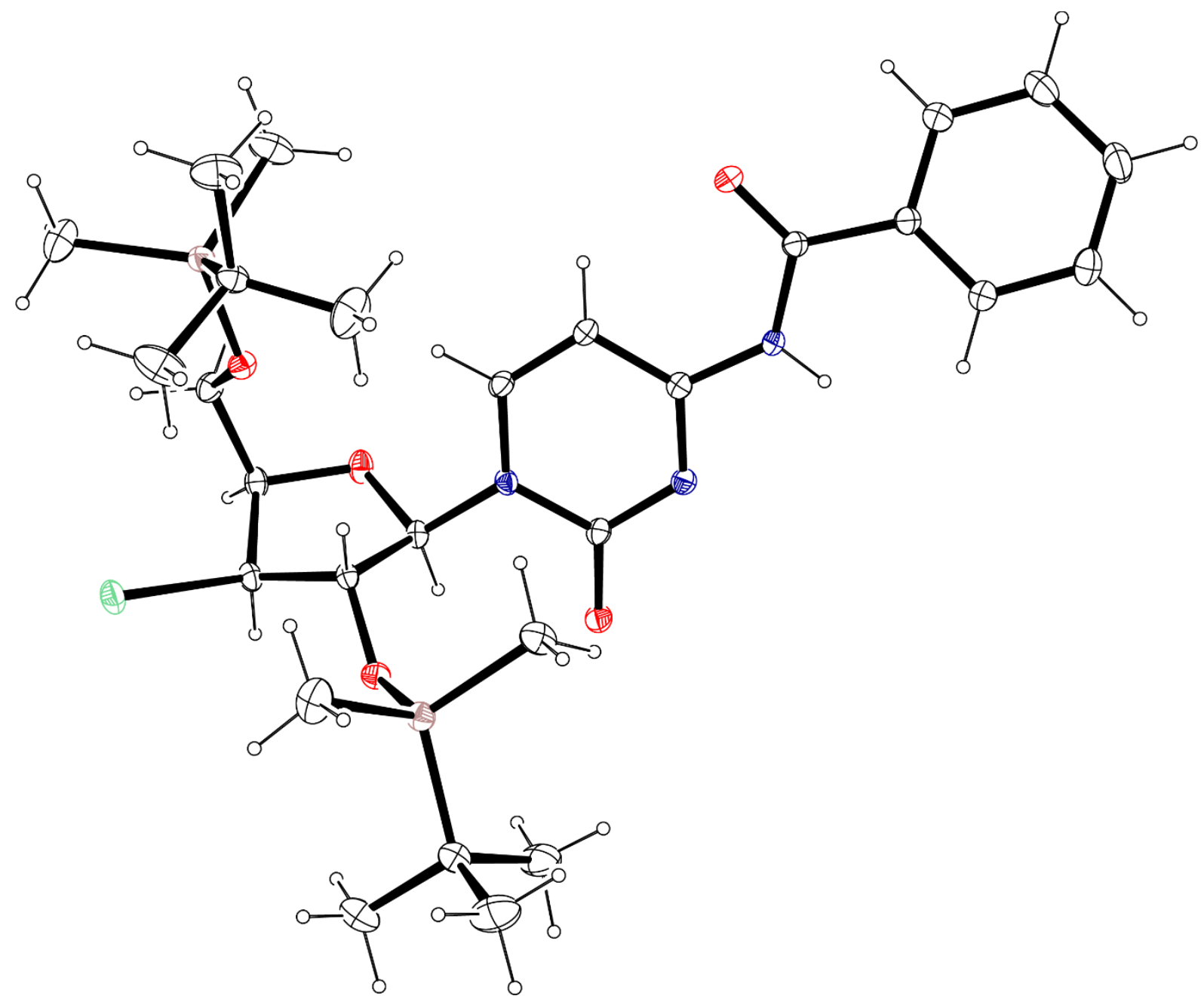

Figure S1. ORTEP diagram of iodide $\mathbf{1 1}$ shown with $30 \%$ probability ellipsoids. 
Table S1. Crystal data and structure refinement for iodide $\mathbf{1 1 .}$

Empirical formula

Formula weight

Temperature

Wavelength

Crystal system

Space group

Unit cell dimensions

Volume

$\mathrm{Z}$

Density (calculated)

Absorption coefficient

$\mathrm{F}(000)$

Crystal size

Theta range for data collection

Index ranges

Reflections collected

Independent reflections

Completeness to theta $=67.684^{\circ}$

Refinement method

Data / restraints / parameters

Goodness-of-fit on $\mathrm{F}^{2}$

Final R indices [I $>2 \operatorname{sigma}(\mathrm{I})]$

$\mathrm{R}$ indices (all data)

Absolute structure parameter

Largest diff. peak and hole
$\mathrm{C}_{28} \mathrm{H}_{44} \mathrm{I} \mathrm{N}_{3} \mathrm{O}_{5} \mathrm{Si}_{2}$

685.74

120(2) K

$1.54184 \AA$

Monoclinic

$P 2_{1}$

$\mathrm{a}=14.84118(14) \AA \quad \alpha=90^{\circ}$

$\mathrm{b}=7.79130(7) \AA \quad \beta=111.8656(10)^{\circ}$

$\mathrm{c}=16.12576(14) \AA \quad \gamma=90^{\circ}$

1730.51(3) $\AA^{3}$

2

$1.316 \mathrm{Mg} / \mathrm{m}^{3}$

$8.232 \mathrm{~mm}^{-1}$

708

$0.682 \times 0.066 \times 0.046 \mathrm{~mm}^{3}$

5.110 to $73.432^{\circ}$

$-18<=\mathrm{h}<=18$

$-8<=\mathrm{k}<=9$

$-19<=1<=20$

24396

$6233[\mathrm{R}(\mathrm{int})=0.0314]$

$99.9 \%$

Full-matrix least-squares on $\mathrm{F}^{2}$

6233 / 1 / 365

1.036

$\mathrm{R} 1=0.0204, \mathrm{wR} 2=0.0508$

$\mathrm{R} 1=0.0212, \mathrm{wR} 2=0.0512$

$-0.022(2)$

0.529 and -0.424 e. $\AA^{-3}$ 
${ }^{1} \mathbf{H},{ }^{13} \mathbf{C}\left\{{ }^{1} \mathbf{H}\right\}$ and ${ }^{31} \mathbf{P}\left\{{ }^{1} \mathbf{H}\right\}$ NMR Spectra of Novel Compounds

4-N-Benzoyl-2'-O-(tert-butyldimethylsilyl)-3'-deoxy-3', $4^{\prime}$-didehydrocytidine-5' -aldehyde (8) - ${ }^{1} \mathrm{H}$ NMR (500 MHz, CDCl 3 )
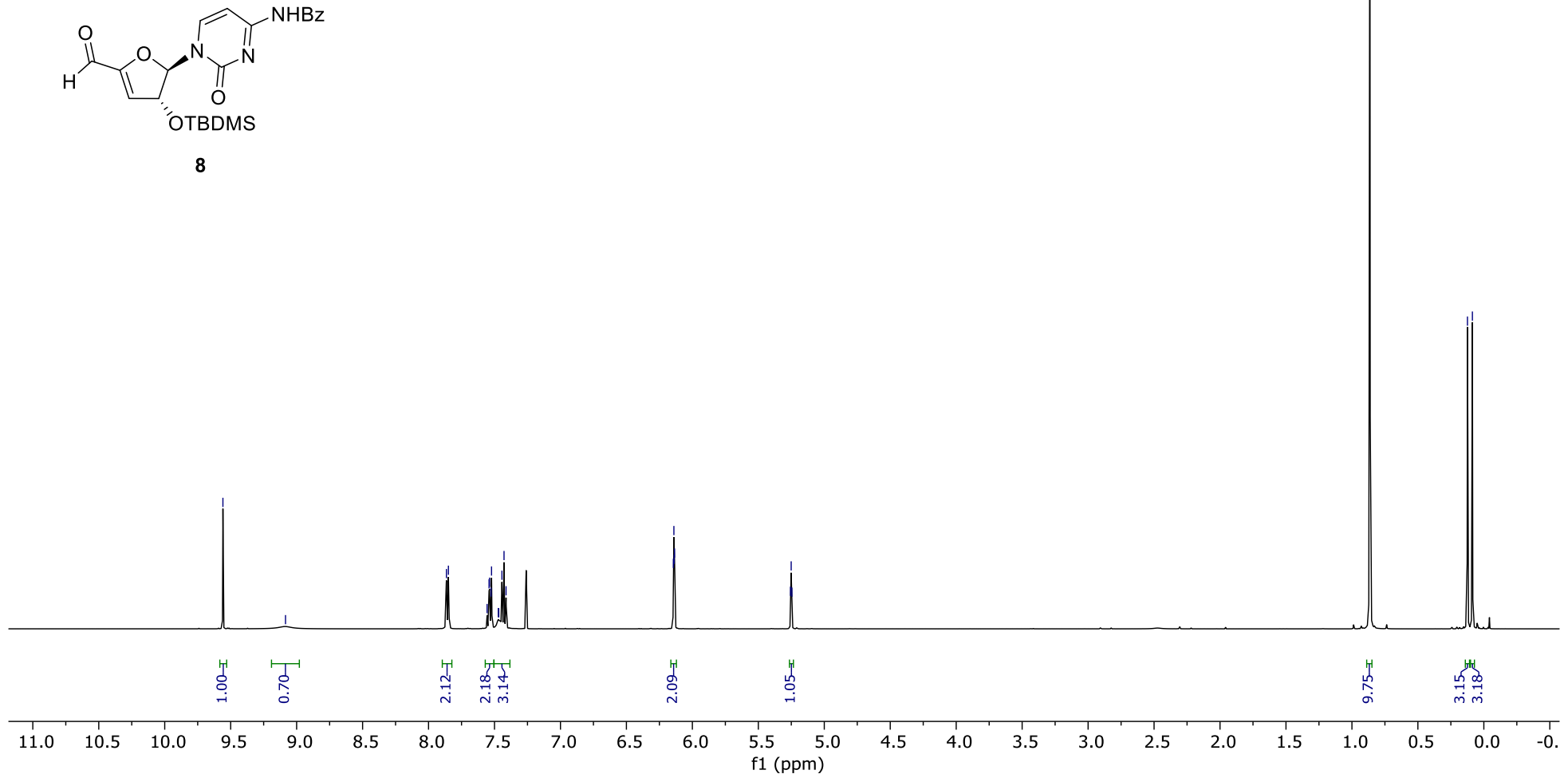
4-N-Benzoyl-2'-O-(tert-butyldimethylsilyl)-3'-deoxy-3',4'-didehydrocytidine-5'-aldehyde (8) - ${ }^{13} \mathrm{C}\left\{{ }^{1} \mathrm{H}\right\} \mathrm{NMR}\left(126 \mathrm{MHz}, \mathrm{CDCl}{ }_{3}\right)$
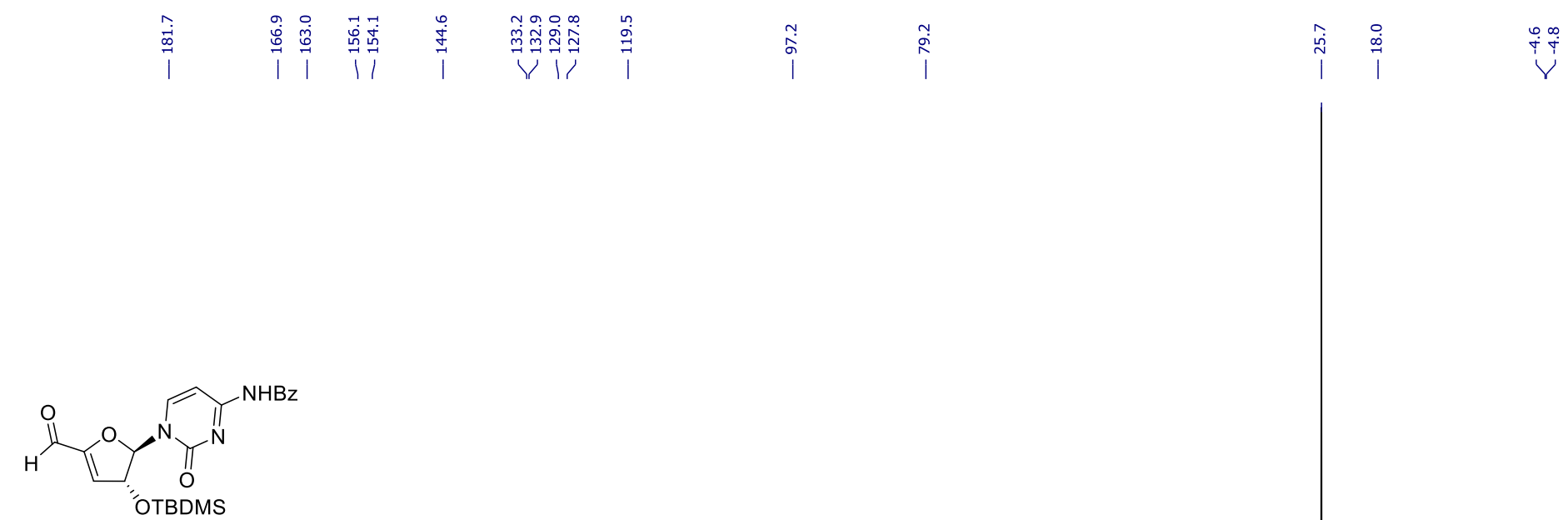

8

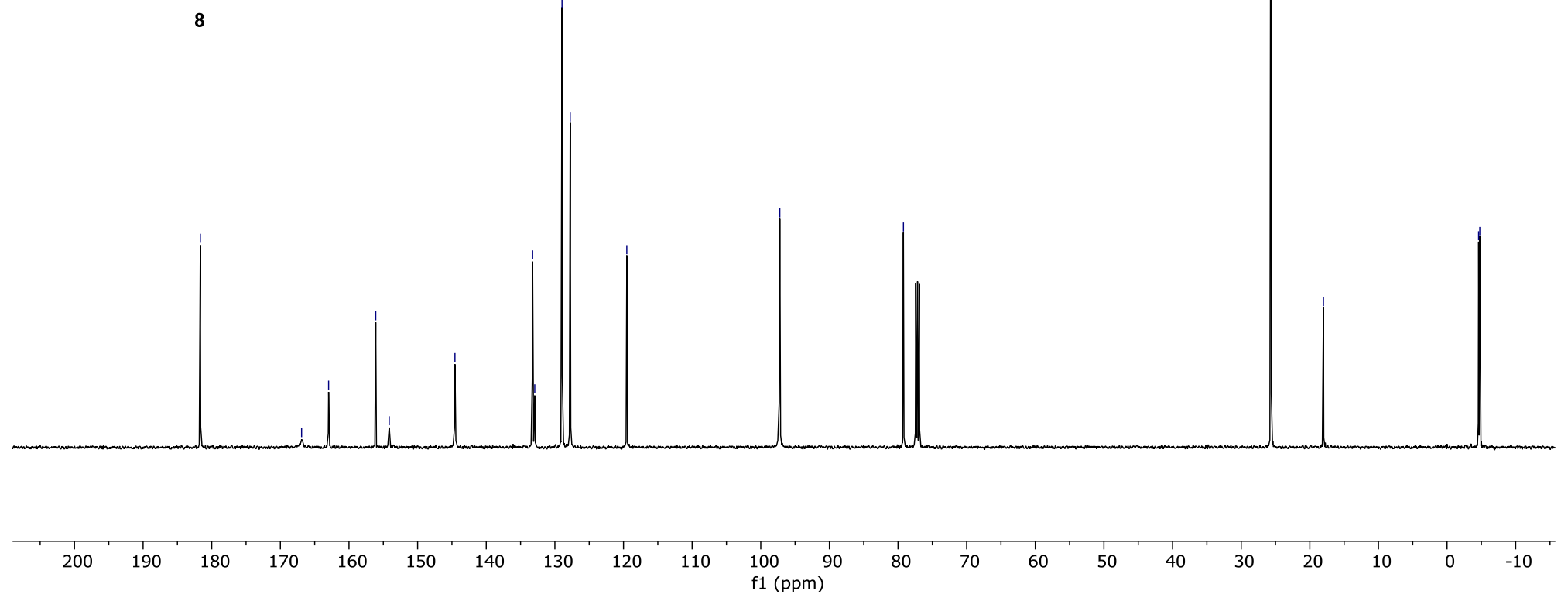


4-N-Benzoyl-2'-O-(tert-butyldimethylsilyl)-3'-deoxy-3', $4^{\prime}$-didehydrocytidine (3) - ${ }^{1} \mathrm{H}$ NMR (500 $\left.\mathrm{MHz}^{\prime}, \mathrm{CDCl}_{3}\right)$
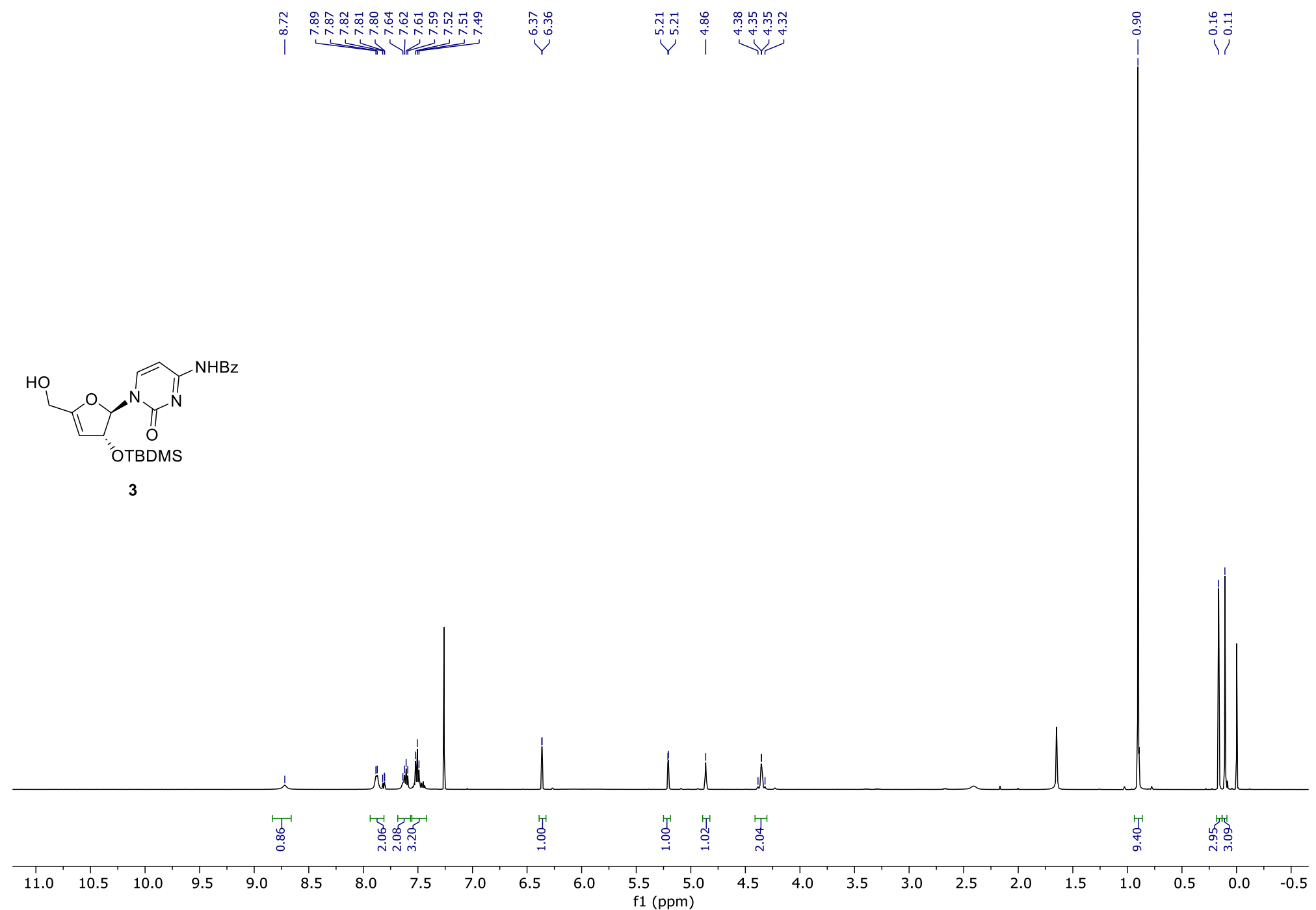
4-N-Benzoyl-2'-O-(tert-butyldimethylsilyl)-3'-deoxy-3', $4^{\prime}$-didehydrocytidine $(3)-{ }^{13} \mathrm{C}\left\{{ }^{1} \mathrm{H}\right\}$ NMR (126 MHz, CDCl 3$)$

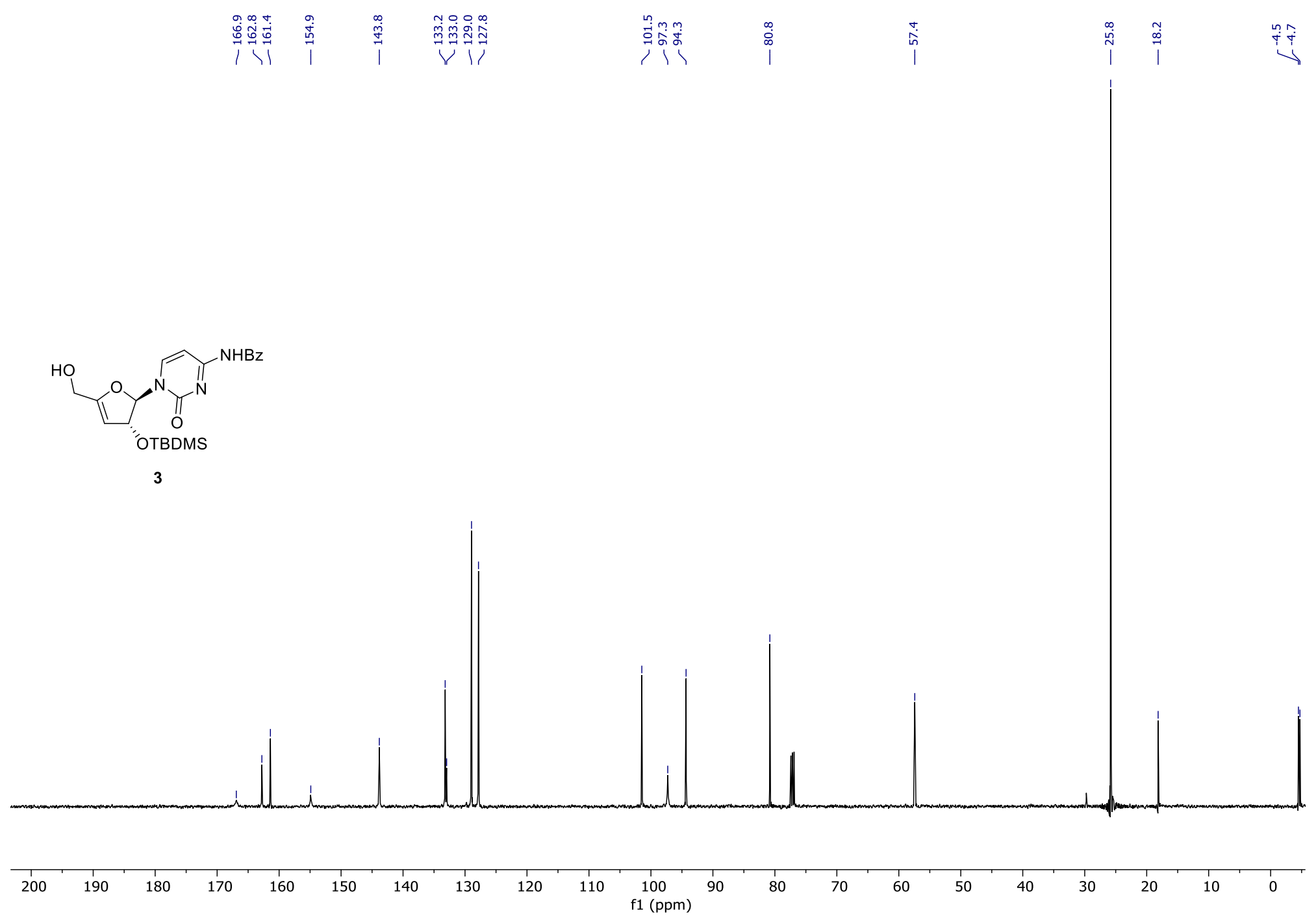


4- $N$-Benzoyl-2',5'-bis- $O$-(tert-butyldimethylsilyl)-3'-deoxy-3',4'-didehydro-5'-(1H-imidazol-1-yl)cytidine (9) - ${ }^{1} \mathrm{H}$ NMR $\left(500 \mathrm{MHz}^{\prime}, \mathrm{CDCl}_{3}\right)$

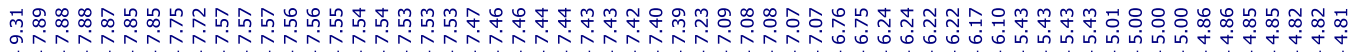

$\underbrace{10}$
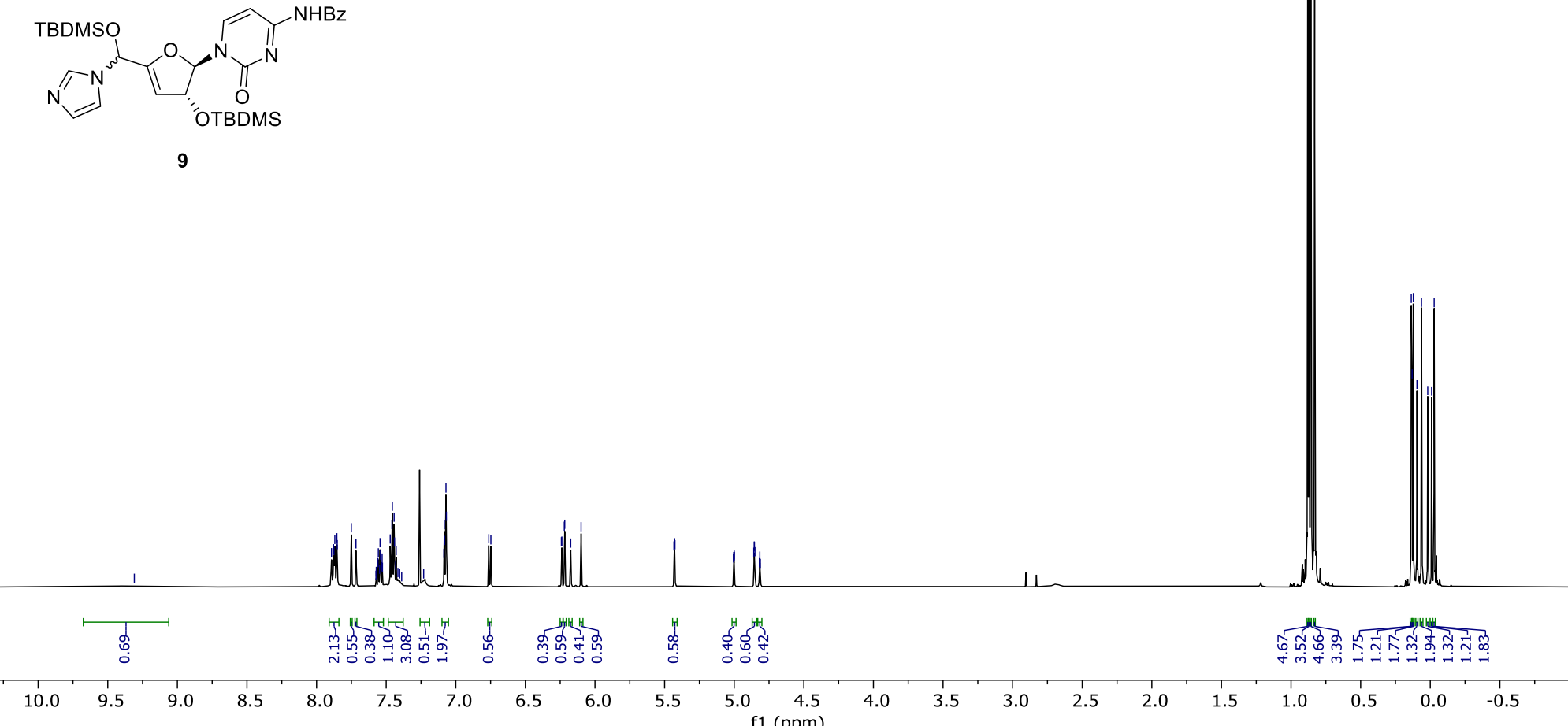


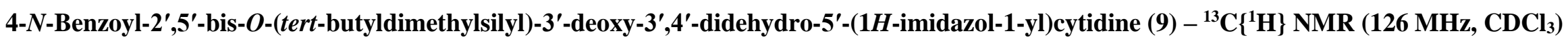

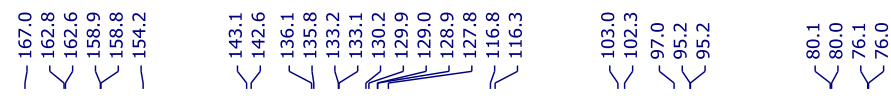

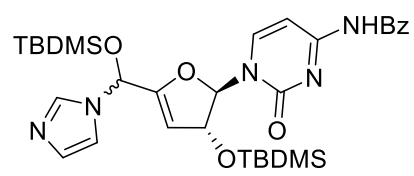

9

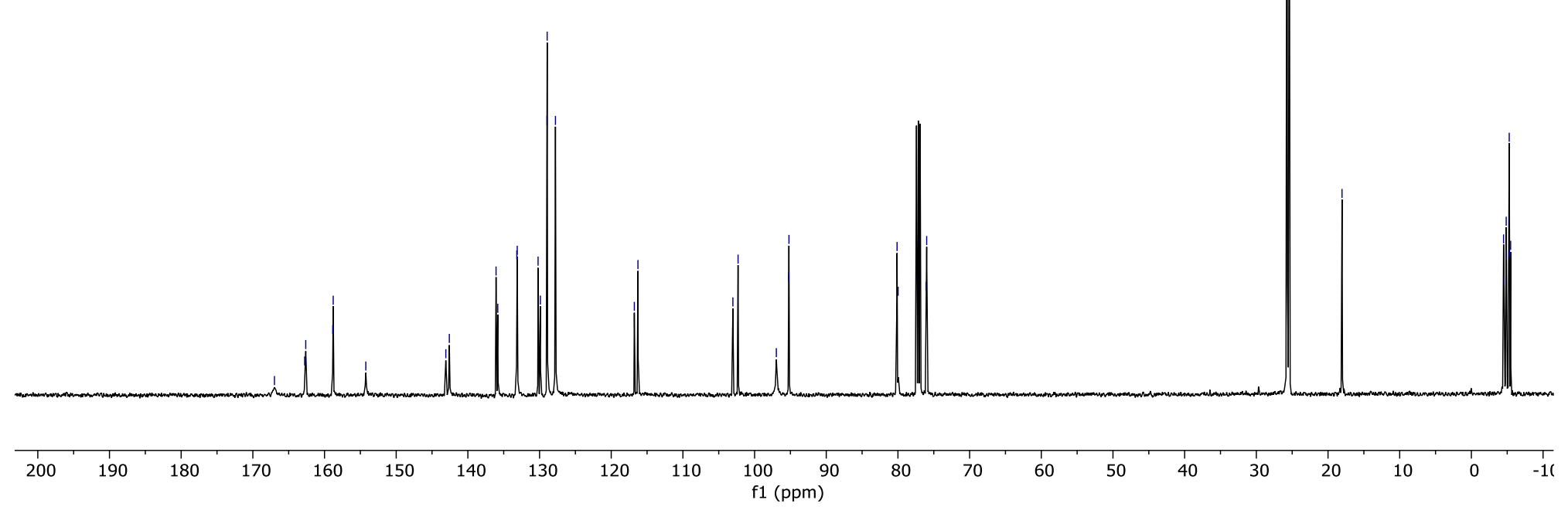


1-(2',5'-bis- $O$-(tert-Butyldimethylsilyl)-3'-iodo- $\beta$-D-threo-pentofuranosyl)-4- $N$-benzoylcytosine (11) - ${ }^{1} \mathrm{H}$ NMR (500 MHz, CDCl 3$)$

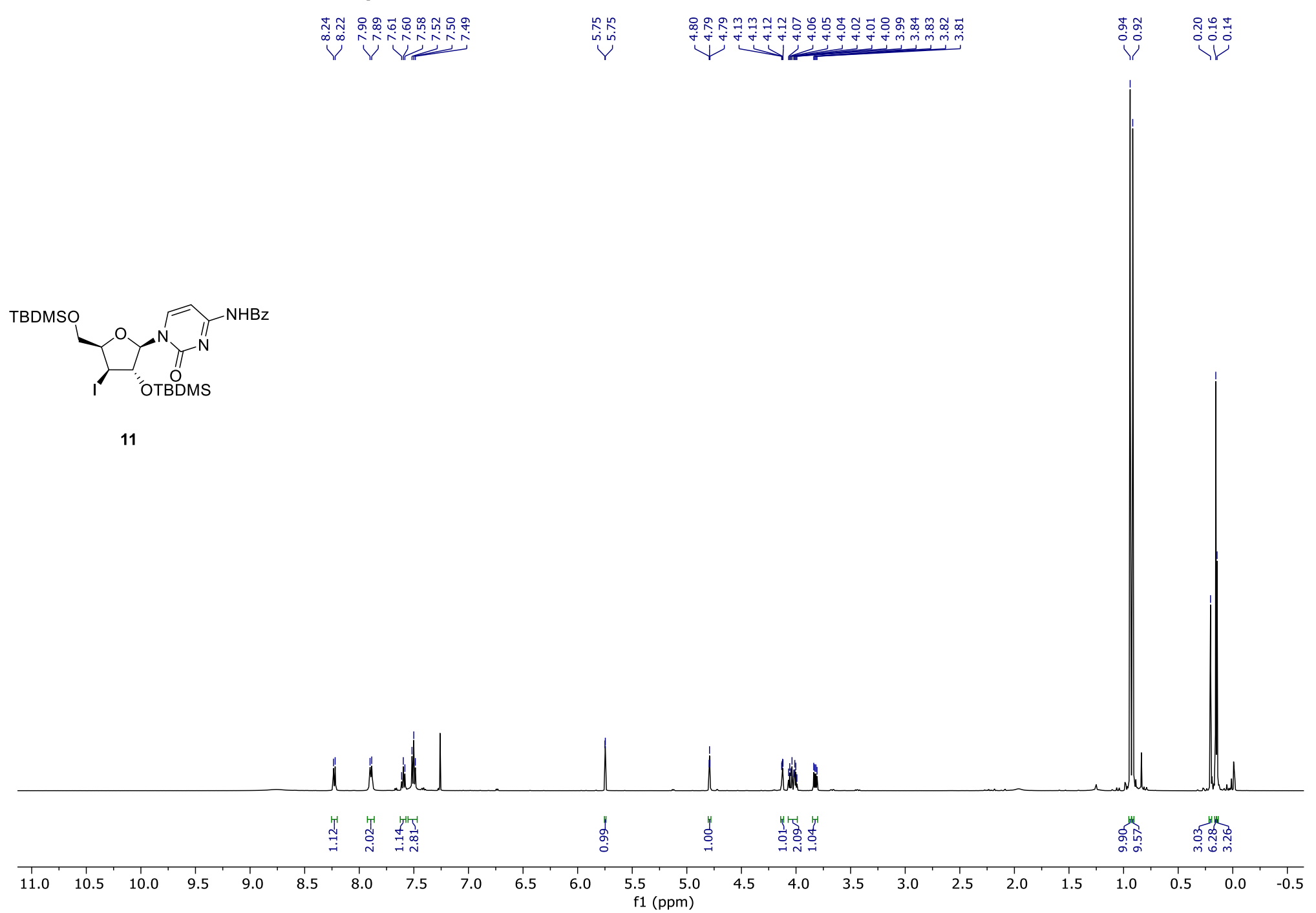


1-(2',5'-bis- $O$-(tert-Butyldimethylsilyl)-3'-iodo- $\beta$-D-threo-pentofuranosyl)-4- $N$-benzoylcytosine $(11)-{ }^{13} \mathrm{C}\left\{{ }^{1} \mathrm{H}\right\}$ NMR $(126 \mathrm{MHz}, \mathrm{CDCl} 3)$

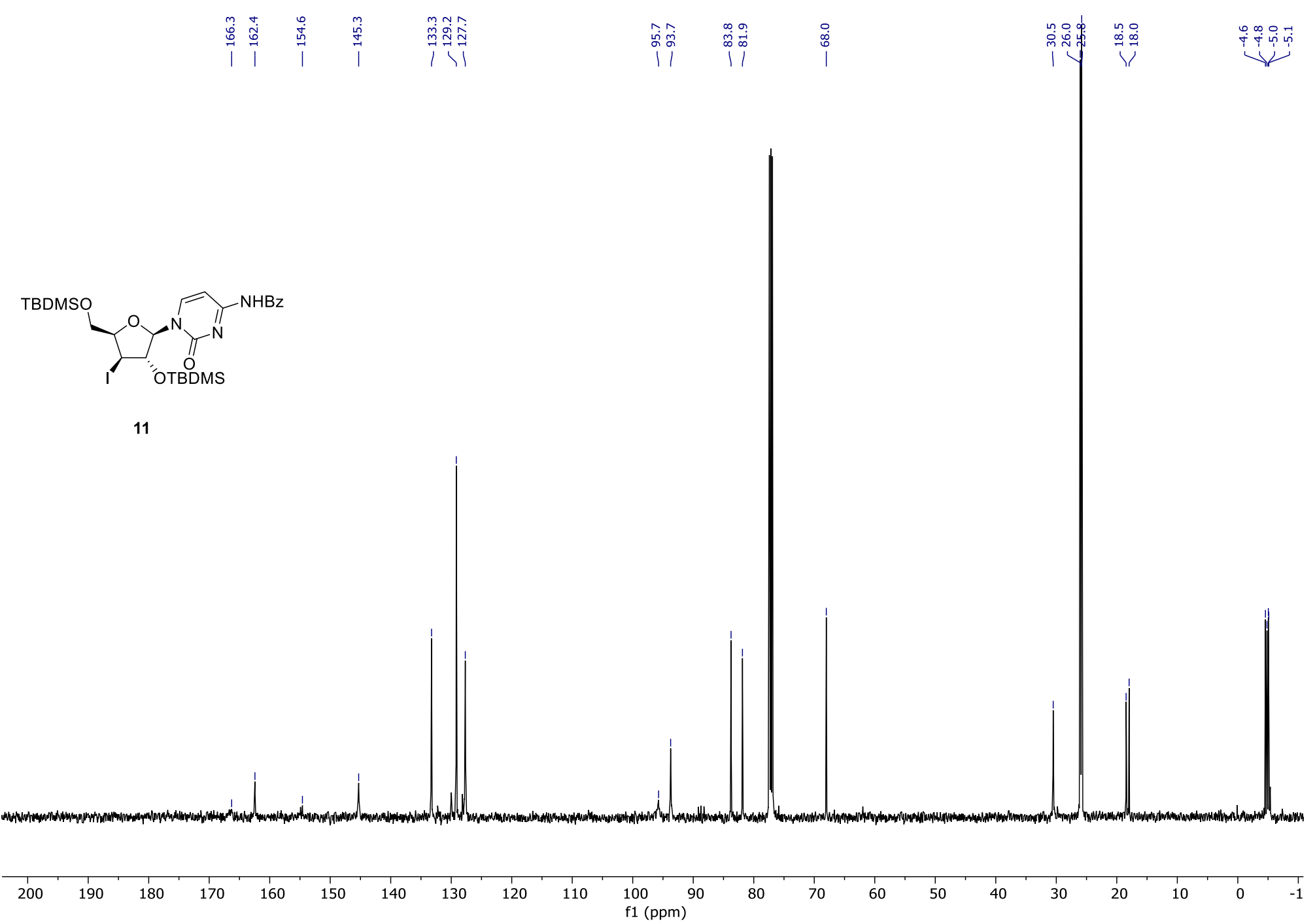


1-(2'-O-(tert-Butyldimethylsilyl)-3'-iodo- $\beta$-D-threo-pentofuranosyl)-4- $N$-benzoylcytosine (12) - $\left.{ }^{1} \mathrm{H} \mathrm{NMR} \mathrm{(500} \mathrm{MHz,} \mathrm{CDCl}_{3}\right)$

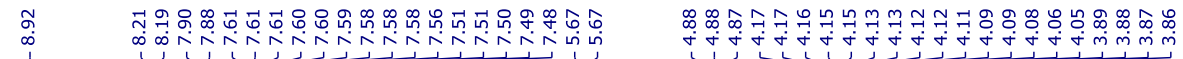

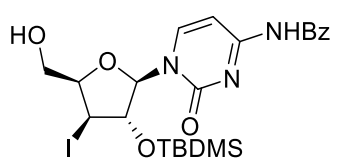

12
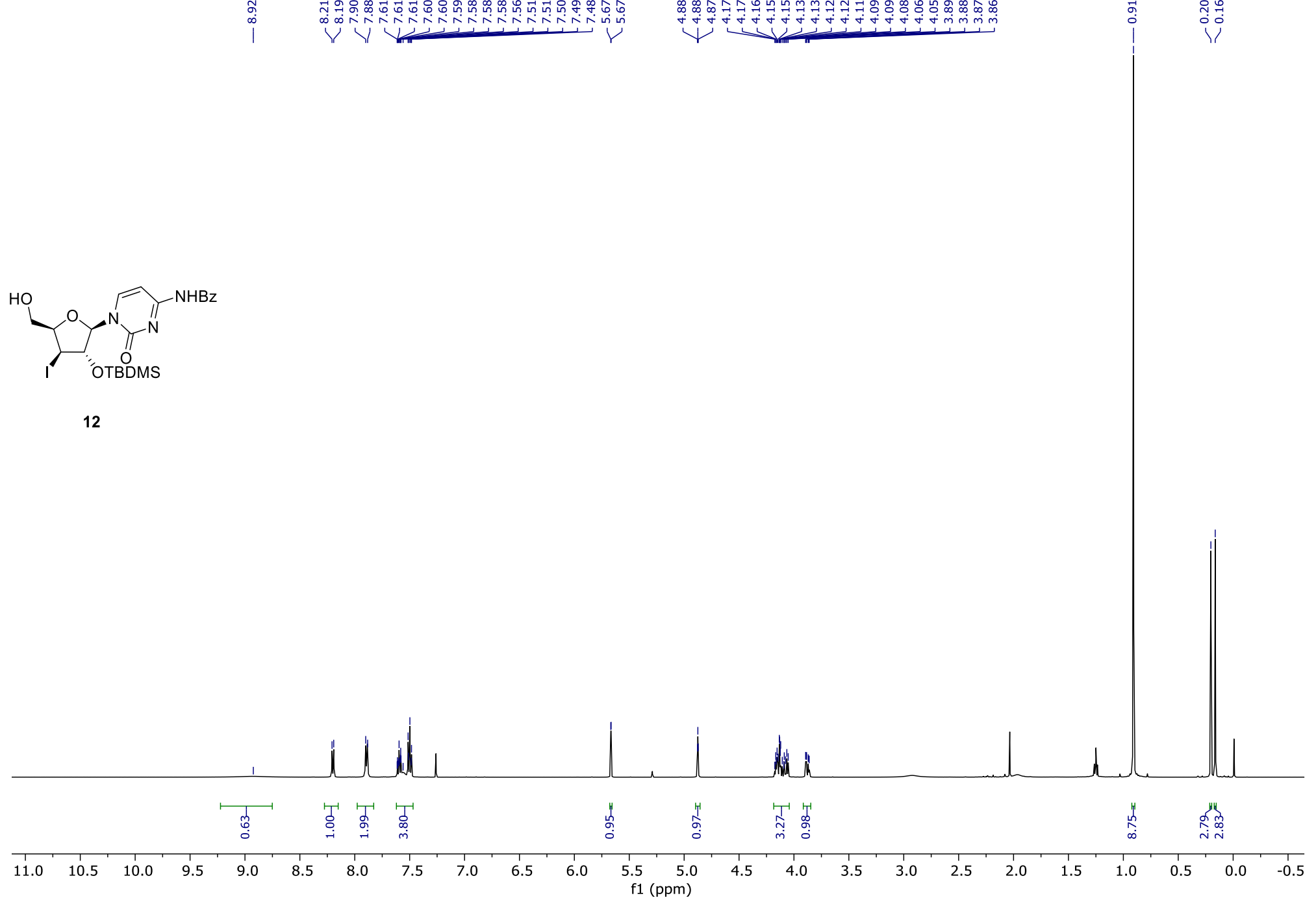
1-(2'-O-(tert-Butyldimethylsilyl)-3'-iodo- $\beta$-D-threo-pentofuranosyl)-4- $N$-benzoylcytosine (12) - ${ }^{13} \mathrm{C}\left\{{ }^{1} \mathrm{H}\right\} \mathrm{NMR}(126 \mathrm{MHz}, \mathrm{CDCl} 3)$

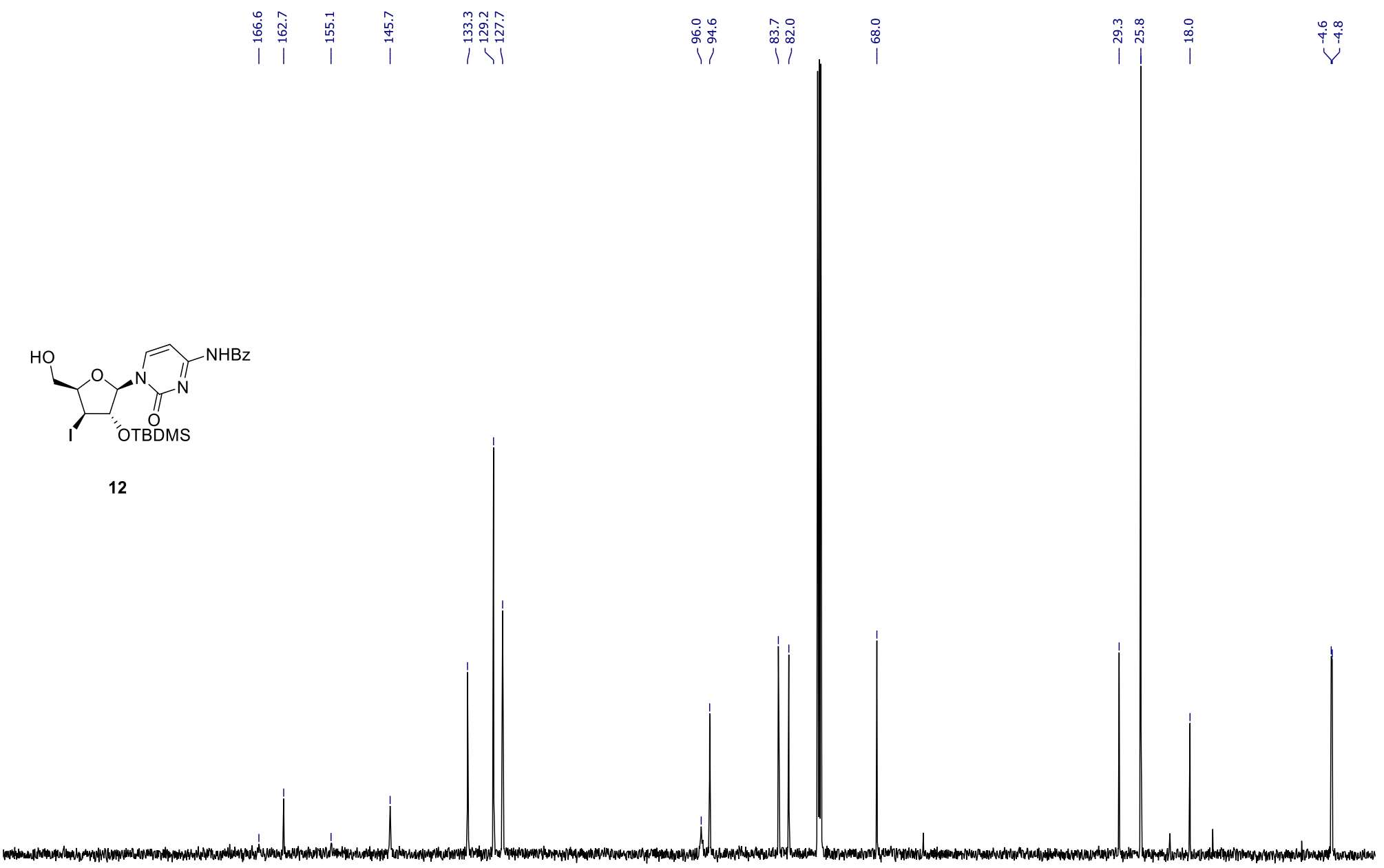

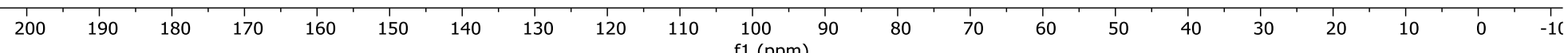


Difluorenylmethyl-4- $N$-benzoyl-2'-O-(tert-butyldimethylsilyl)-3'-deoxy-3', $4^{\prime}$-didehydrocytidine-5'-phosphate (13) - ${ }^{1} \mathbf{H}^{\mathrm{NMR}}$ (500 MHz, CDCl )
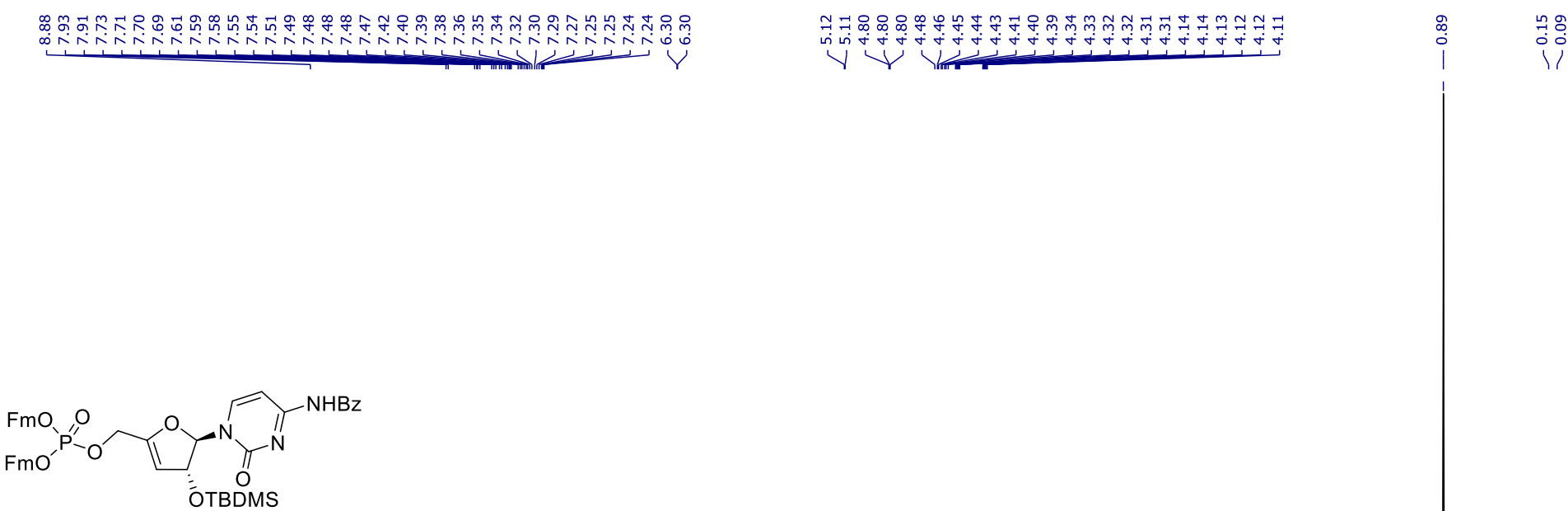

13

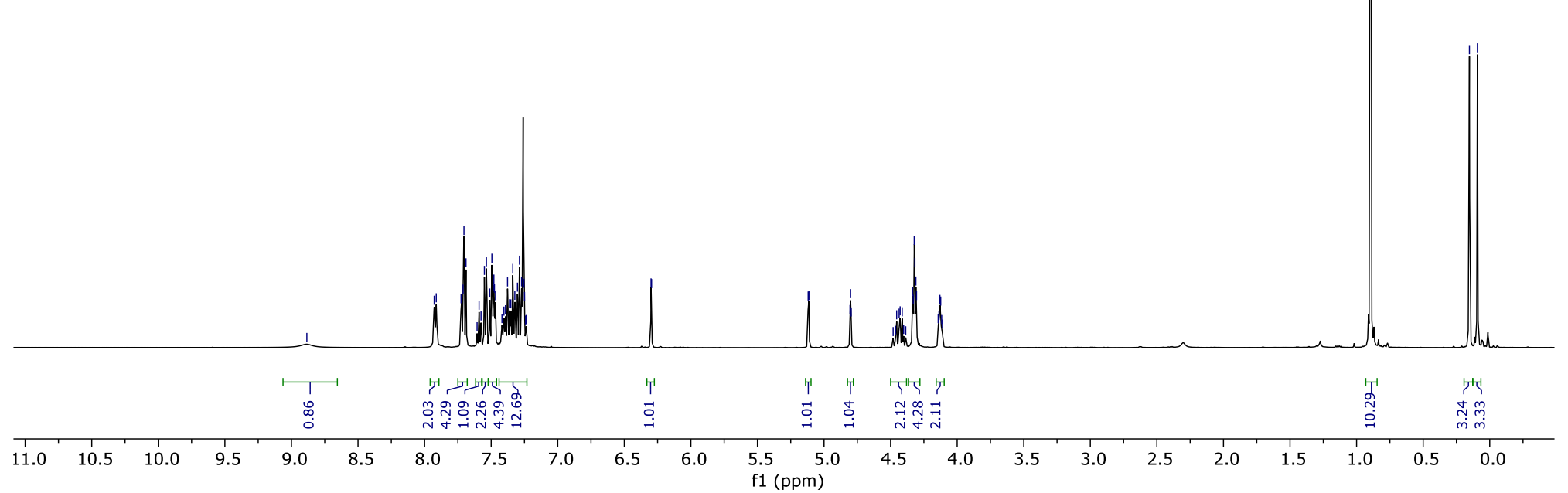


Difluorenylmethyl-4- $N$-benzoyl-2'-O-(tert-butyldimethylsilyl)-3'-deoxy-3', $4^{\prime}$-didehydrocytidine-5'-phosphate (13) - ${ }^{13} \mathrm{C}\left\{{ }^{1} \mathbf{H}^{\prime}\right.$ NMR (126 $\left.\mathrm{MHz}, \mathrm{CDCl}_{3}\right)$

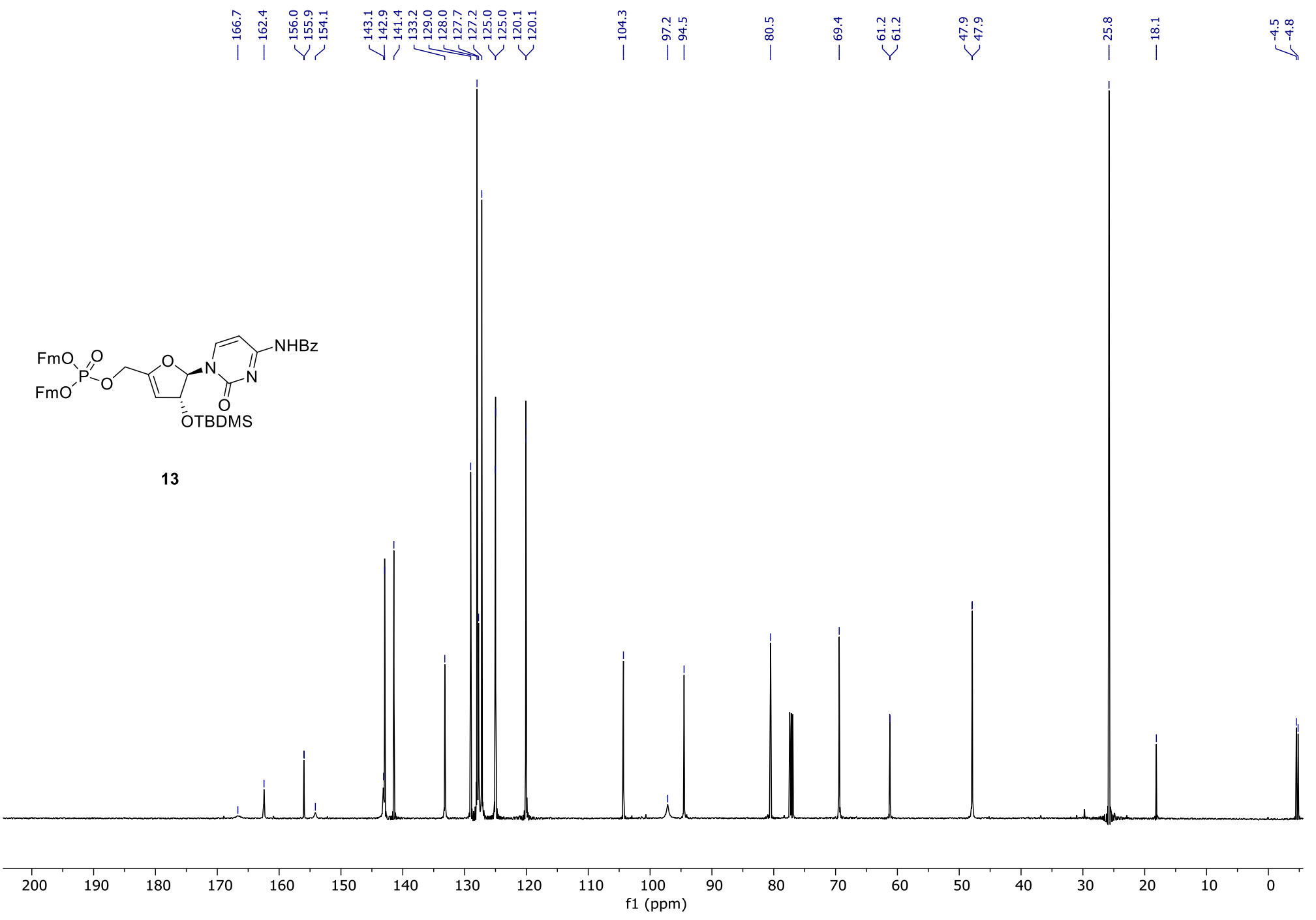


Difluorenylmethyl-4- $N$-benzoyl-2'-O-(tert-butyldimethylsilyl)-3'-deoxy-3', $4^{\prime}$-didehydrocytidine-5' - phosphate $(13)-{ }^{31} \mathbf{P}\left\{{ }^{1} \mathrm{H}\right\}$ NMR $(202 \mathrm{MHz}, \mathrm{CDCl} 3)$

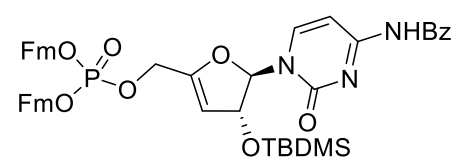

13 
2'-O-(tert-Butyldimethylsilyl)-3'-deoxy-3',4'-didehydrocytidine-5'-phosphate triethylammonium salt (14) - ${ }^{1} \mathrm{H}$ NMR (500 MHz, CD $\left.{ }_{3} \mathrm{OD}\right)$

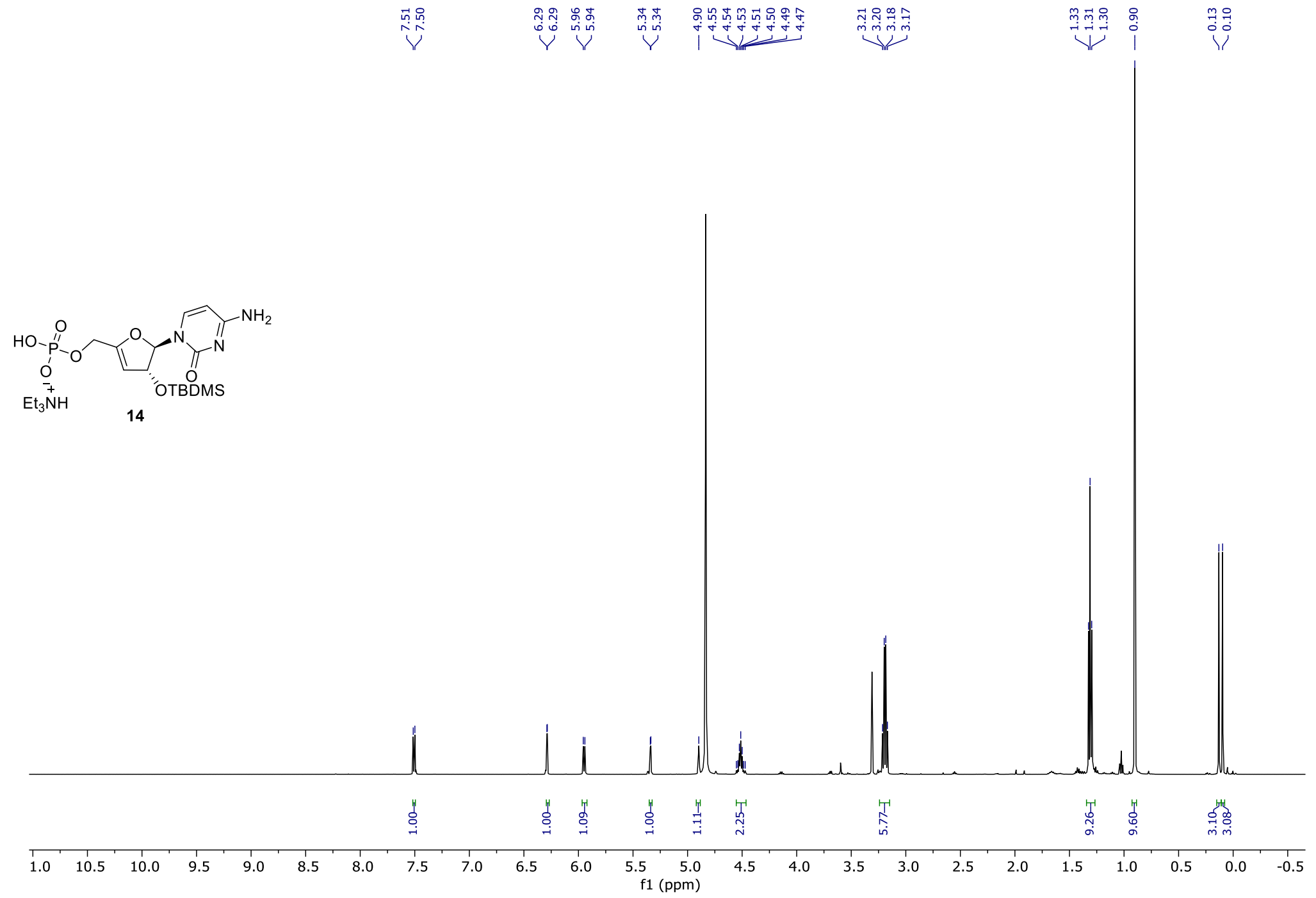


$2^{\prime}$-O-(tert-Butyldimethylsilyl)-3'-deoxy-3',4'-didehydrocytidine-5'-phosphate triethylammonium salt (14) - ${ }^{13} \mathrm{C}\left\{{ }^{1} \mathrm{H}\right\} \mathrm{NMR}\left(126 \mathrm{MHz}, \mathrm{CD}{ }_{3} \mathrm{OD}\right)$

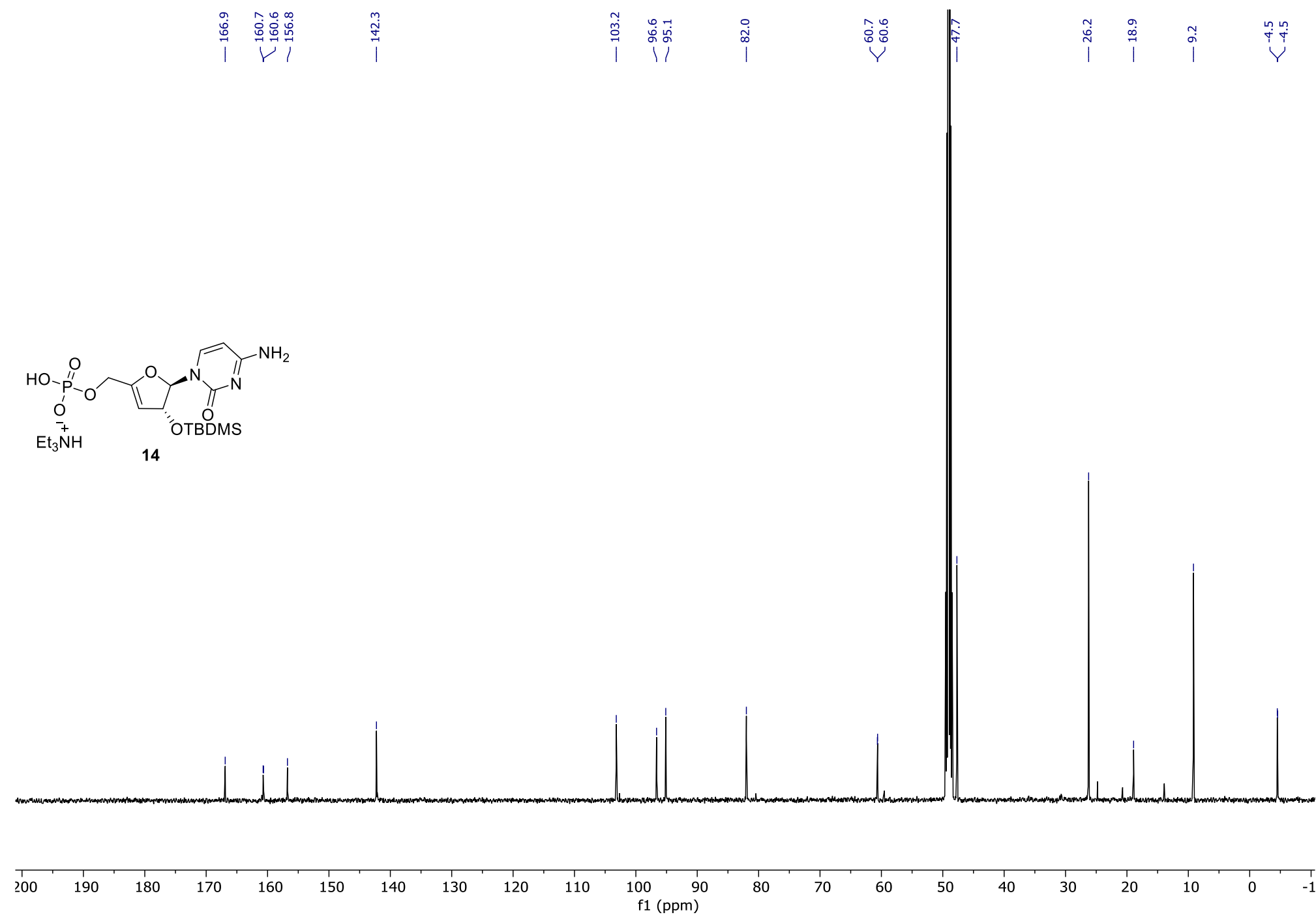


2'-O-(tert-Butyldimethylsilyl)-3'-deoxy-3', $4^{\prime}$-didehydrocytidine-5' -phosphate triethylammonium salt (14) - ${ }^{31} \mathbf{P}\left\{{ }^{1} \mathrm{H}\right\}$ NMR $\left(202 \mathrm{MHz}, \mathrm{CD}{ }_{3} \mathrm{OD}\right)$

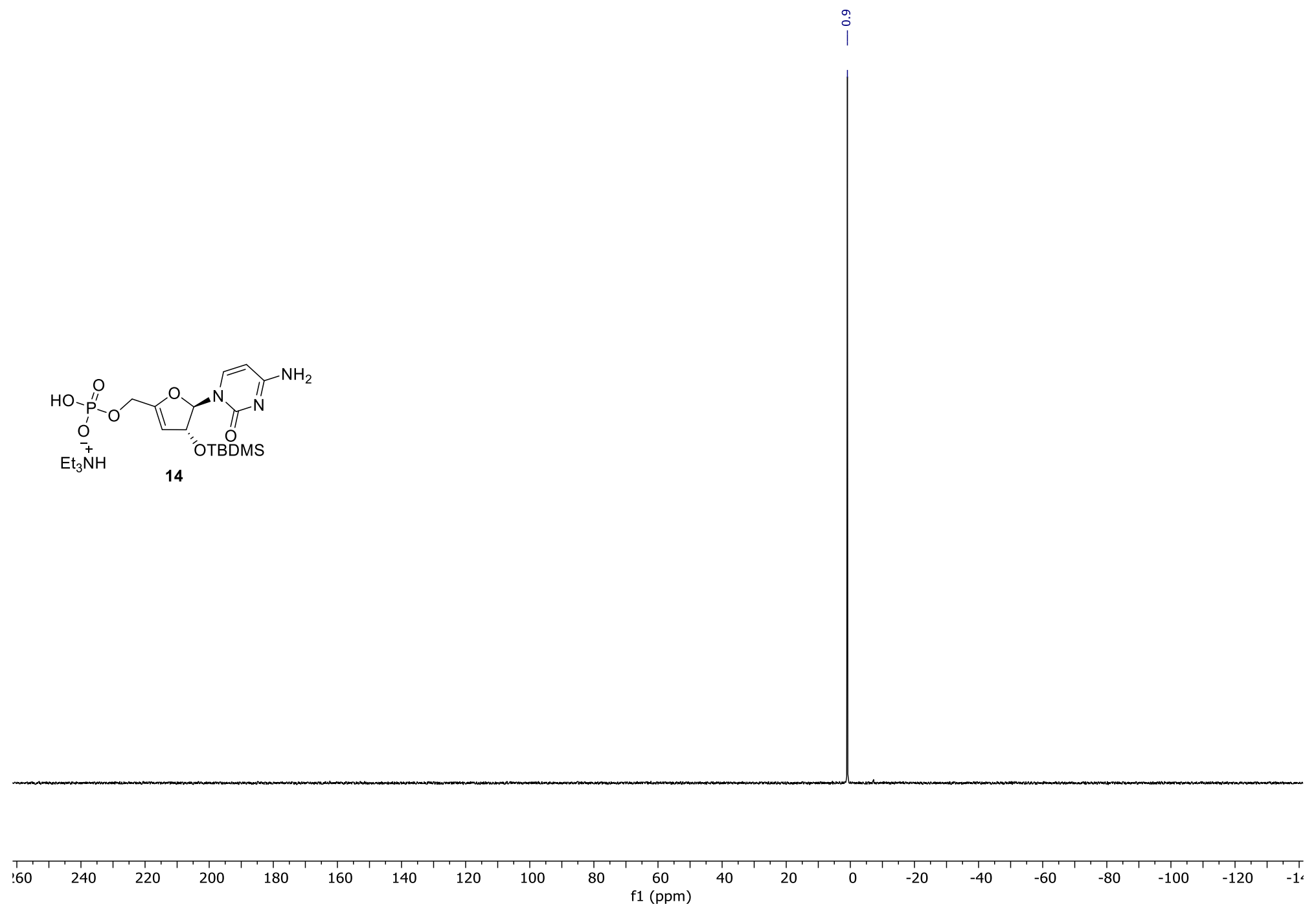


3'-Deoxy-3' ' $^{\prime}$-didehydrocytidine-5'-phosphate sodium salt (ddhCMP, 15) - ${ }^{1}$ H NMR (500 MHz, $\left.\mathrm{D}_{2} \mathrm{O}\right)$
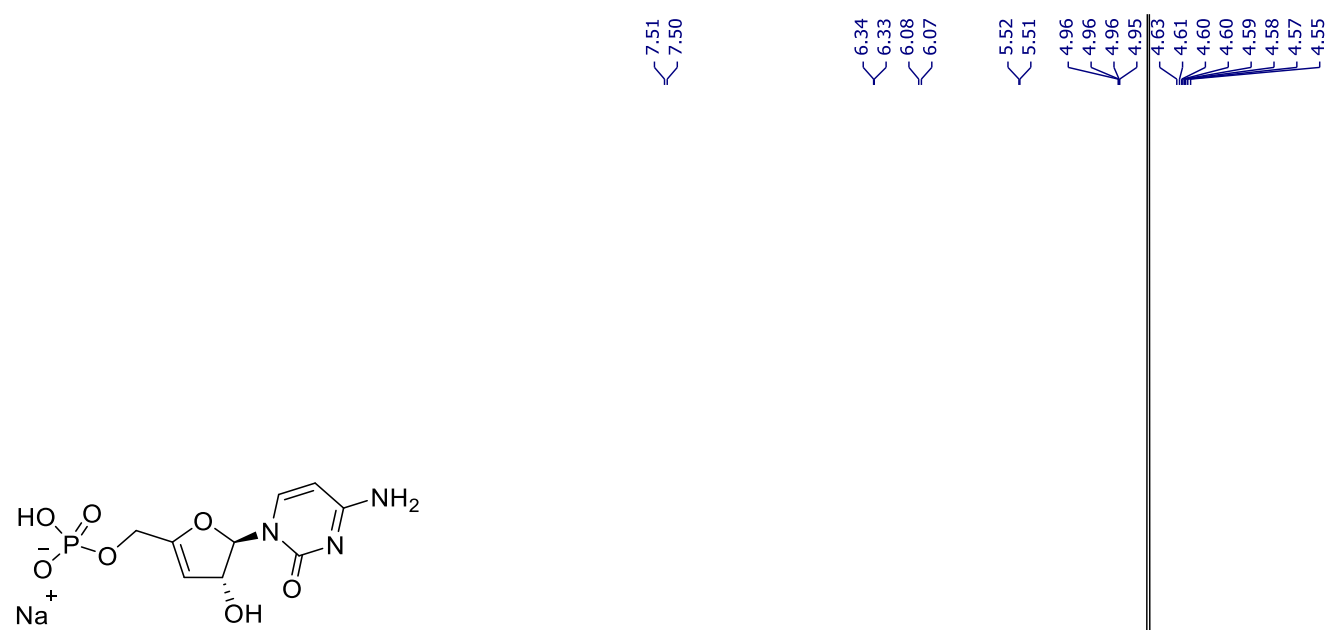

15

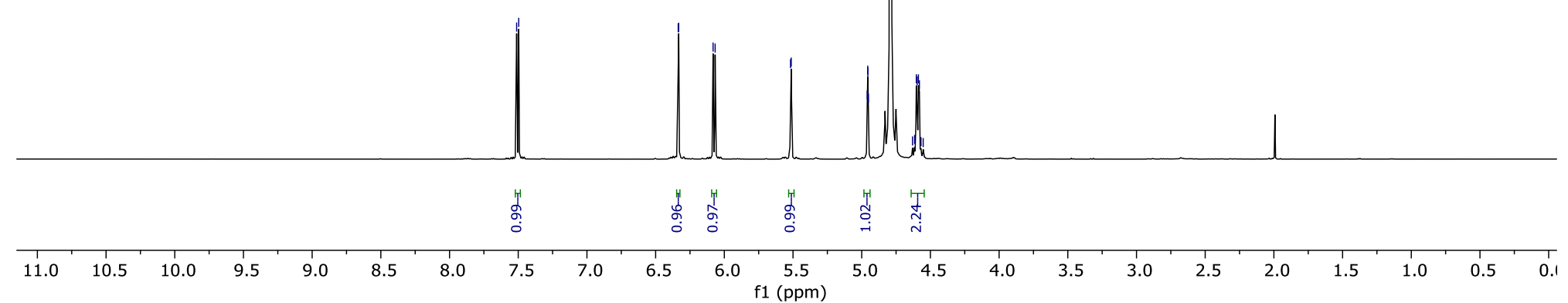


3'-Deoxy-3', $4^{\prime}$-didehydrocytidine-5' -phosphate sodium salt (ddhCMP, 15) - ${ }^{13} \mathrm{C}\left\{{ }^{1} \mathrm{H}\right\}$ NMR (126 MHz, D2O)
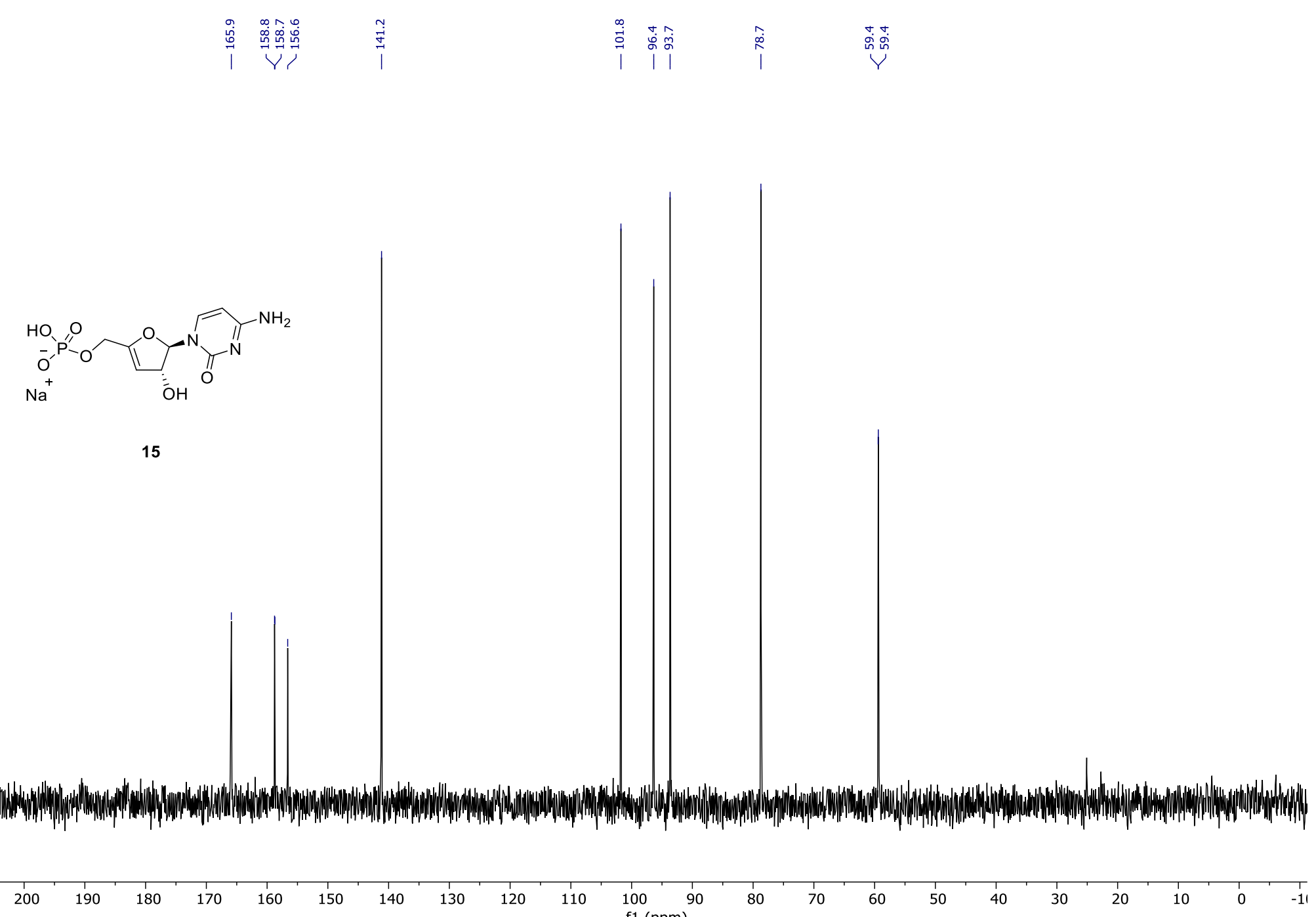
$3^{\prime}$-Deoxy-3' 3' $^{\prime}$-didehydrocytidine-5'-phosphate sodium salt (ddhCMP, 15) - ${ }^{31} \mathrm{P}\left\{{ }^{1} \mathrm{H}\right\}$ NMR $\left(202 \mathrm{MHz}, \mathrm{D}_{2} \mathrm{O}\right)$
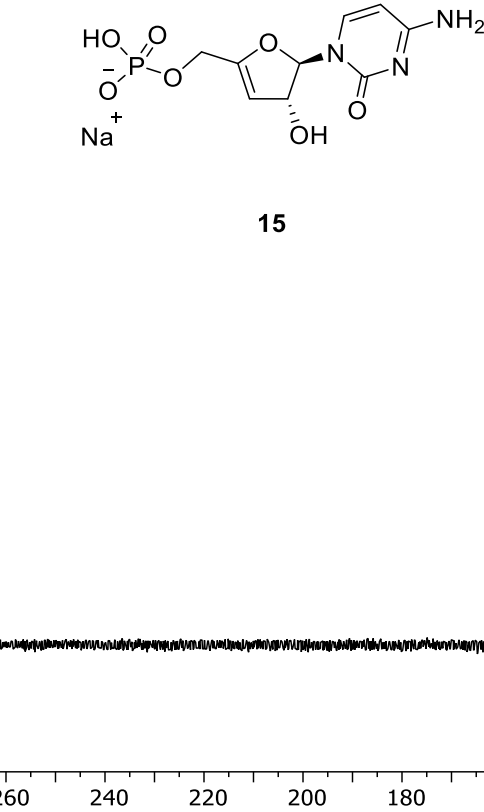

$200 \quad 180$

160

140 
$2^{\prime}$-O-(tert-Butyldimethylsilyl)-3'-deoxy-3',4'-didehydrocytidine-5'-diphosphate bis(triethylammonium) salt (16) - ${ }^{1} \mathrm{H}$ NMR (500 MHz, CD $\left.{ }_{3} \mathrm{OD}\right)$

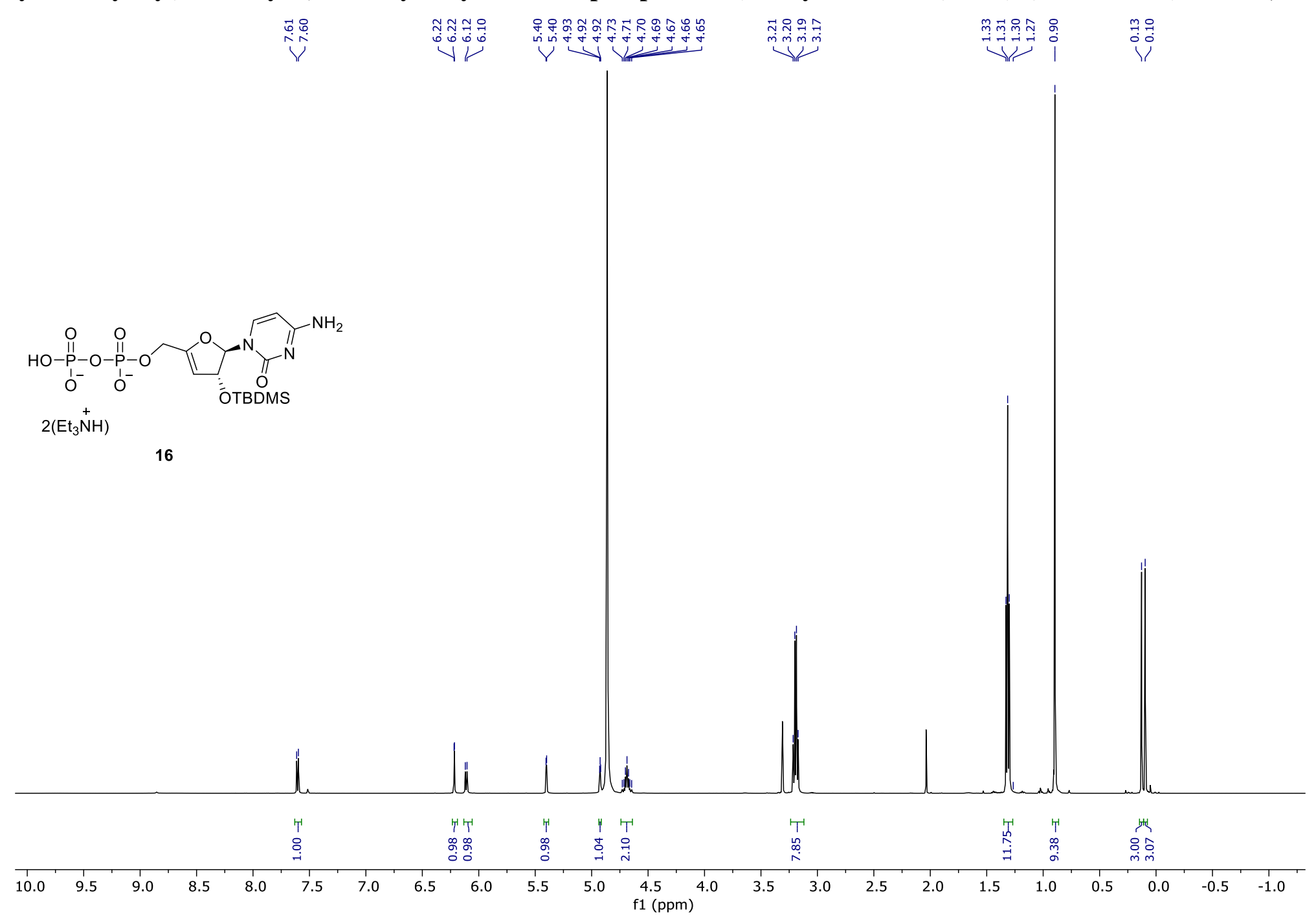


$2^{\prime}$-O-(tert-Butyldimethylsilyl)-3'-deoxy-3',4'-didehydrocytidine-5'-diphosphate bis(triethylammonium) salt (16) - ${ }^{13} \mathrm{C}\left\{{ }^{1} \mathrm{H}\right\}$ NMR $\left(126 \mathrm{MHz}, \mathrm{CD}{ }_{3} \mathrm{OD}\right)$

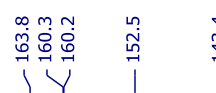

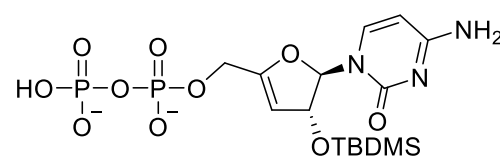

$2\left(\mathrm{Et}_{3} \stackrel{+}{\mathrm{N}} \mathrm{H}\right)$
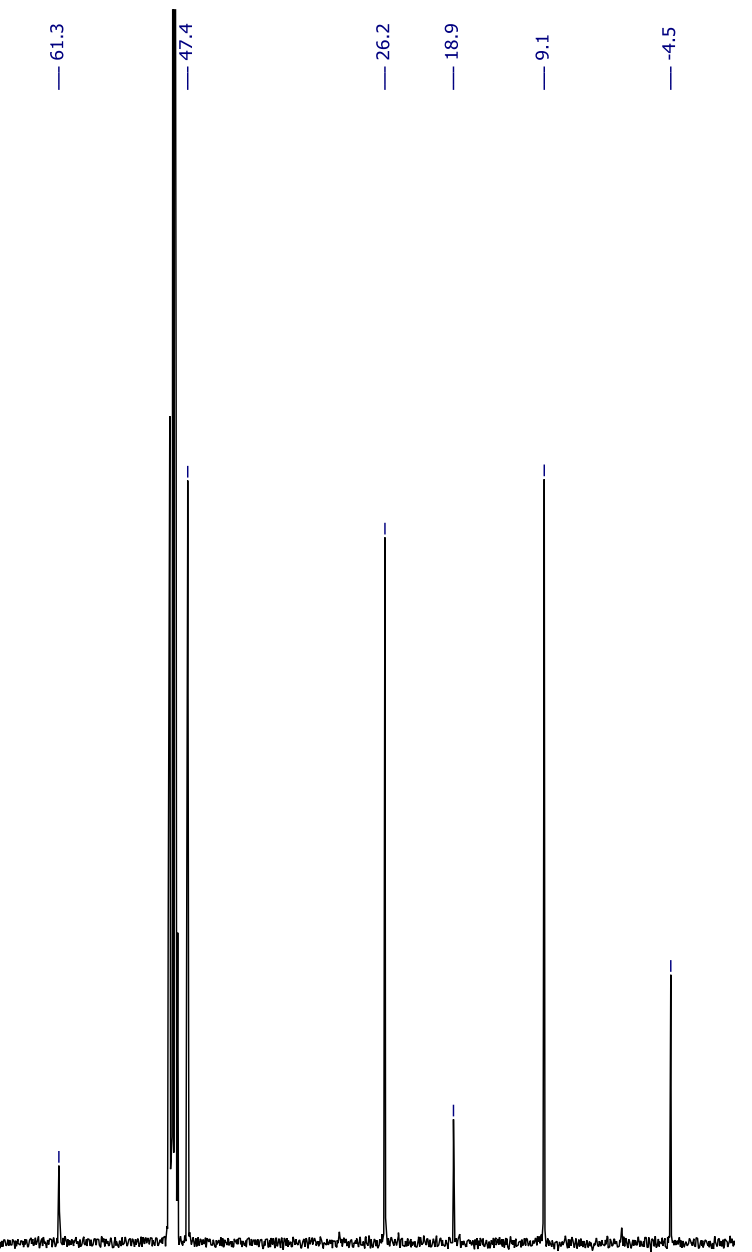
$2^{\prime}$-O-(tert-Butyldimethylsilyl)-3'-deoxy-3',4'-didehydrocytidine-5'-diphosphate bis(triethylammonium) salt (16) - ${ }^{31} \mathbf{P}\left\{{ }^{1} \mathrm{H}\right\}$ NMR $\left(202 \mathrm{MHz}, \mathrm{CD}{ }_{3} \mathrm{OD}\right)$

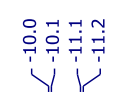

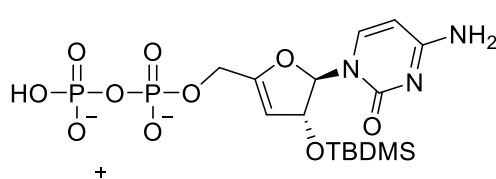

$2\left(\mathrm{Et}_{3} \stackrel{+}{\mathrm{N}} \mathrm{H}\right)$

16

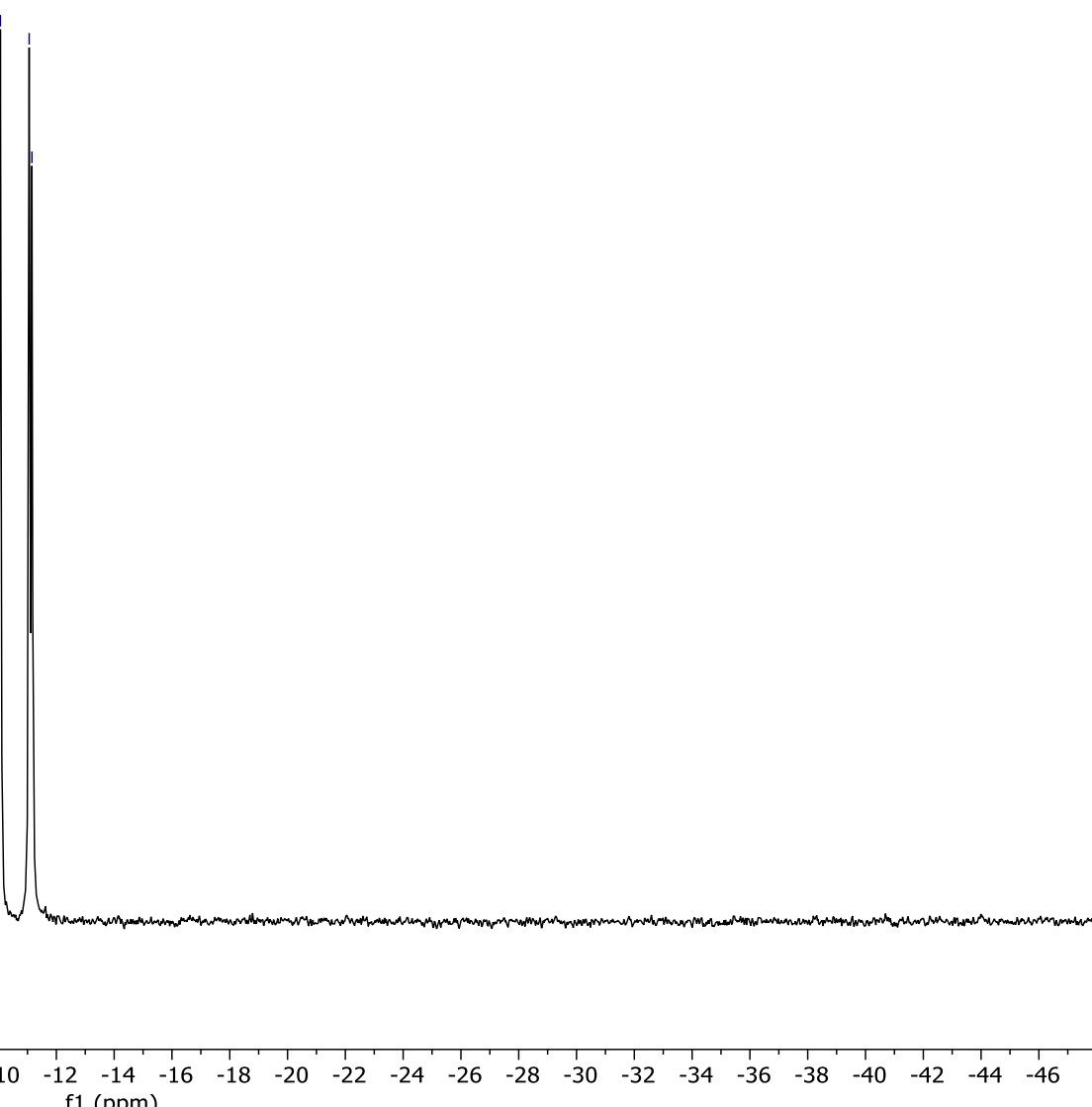

$\begin{array}{rlllllllllllllllllllllll}70 & 18 & 16 & 14 & 12 & 10 & 8 & 6 & 4 & 2 & 0 & -2 & -4 & -6 & -8 & -10 & -12 & -14 & -16 \\ \text { f1 } & (\mathrm{ppm}) & & \end{array}$ 


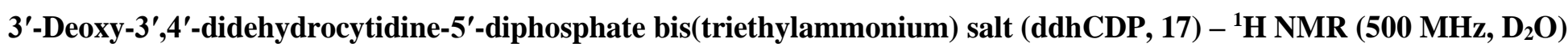

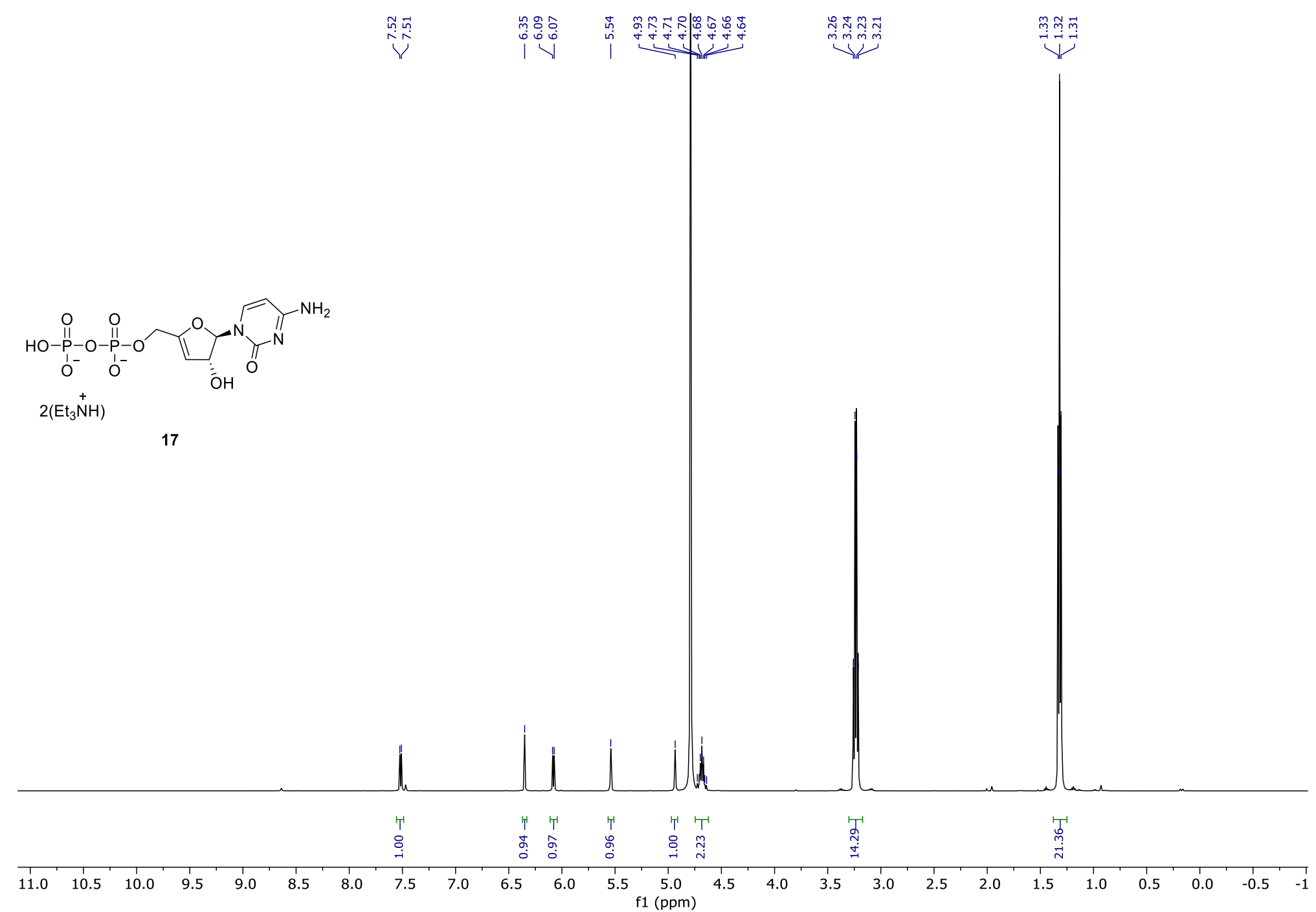


$3^{\prime}$-Deoxy-3', $4^{\prime}$-didehydrocytidine-5'-diphosphate bis(triethylammonium) salt (ddhCDP, 17) $-{ }^{13} \mathrm{C}\left\{{ }^{1} \mathrm{H}\right\} \mathrm{NMR}\left(126 \mathrm{MHz}, \mathrm{D}_{2} \mathrm{O}\right)$

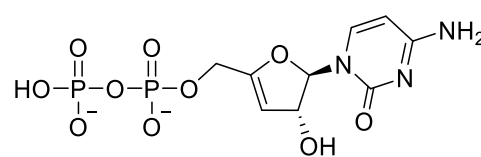

$2\left(\mathrm{Et}_{3} \mathrm{NH}\right)$ 
$3^{\prime}$-Deoxy-3', $4^{\prime}$-didehydrocytidine-5'-diphosphate bis(triethylammonium) salt (ddhCDP, 17) - ${ }^{31} \mathrm{P}\left\{{ }^{1} \mathrm{H}\right\} \mathrm{NMR}\left(202 \mathrm{MHz}, \mathrm{D}_{2} \mathrm{O}\right)$

ำ

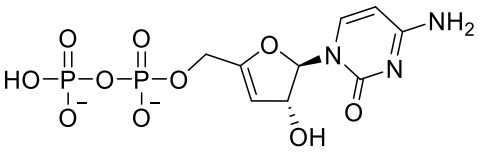

$2\left(\mathrm{Et}_{3} \stackrel{+}{\mathrm{N}} \mathrm{H}\right)$

17 
2'-O-(tert-Butyldimethylsilyl)-3'-deoxy-3',4'-didehydrocytidine-5'-triphosphate tris(triethylammonium) salt (18) - ${ }^{1} \mathrm{H}$ NMR (500 MHz, $\left.\mathrm{D}_{2} \mathrm{O}\right)$

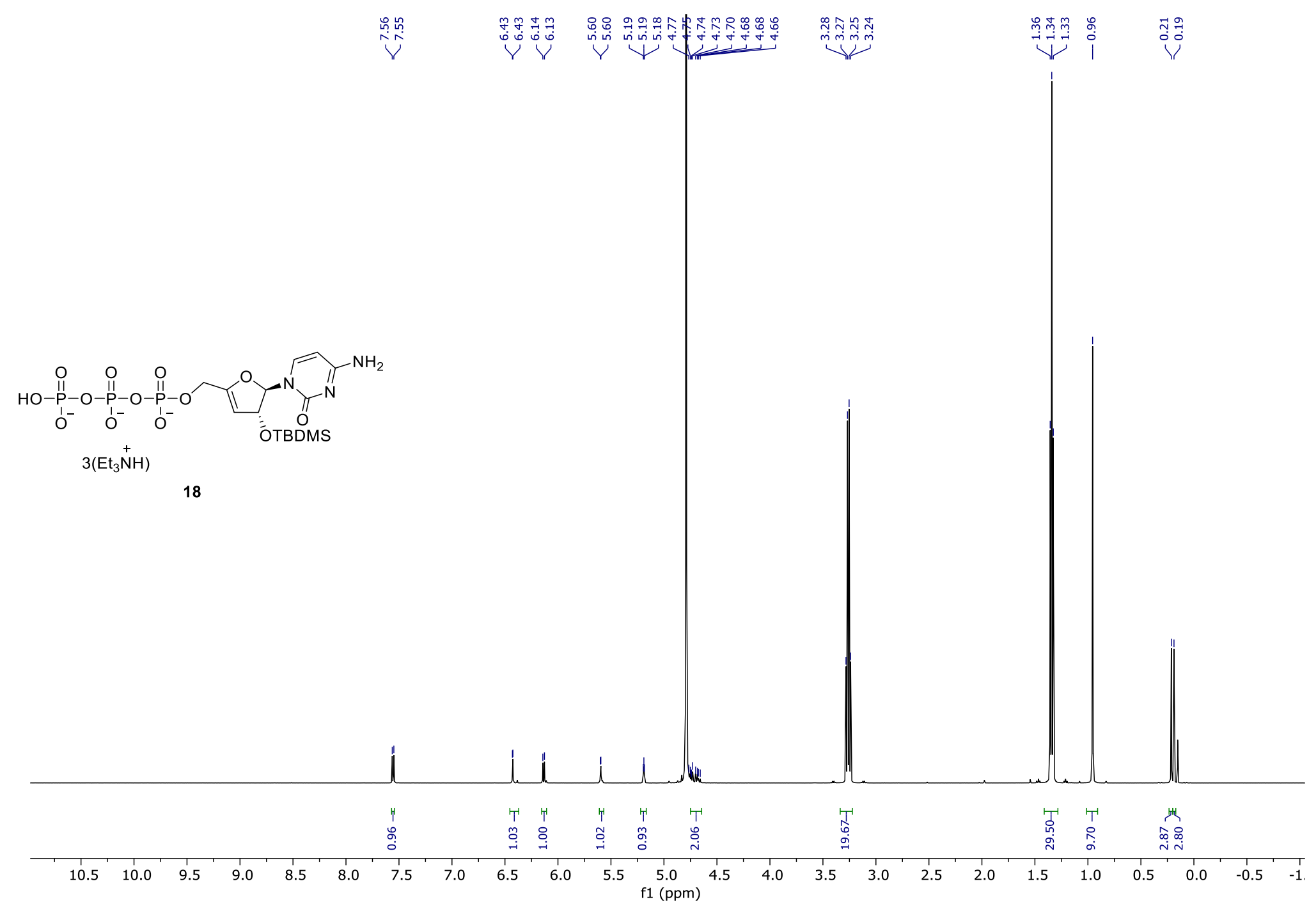


$2^{\prime}$-O-(tert-Butyldimethylsilyl)-3'-deoxy-3', $4^{\prime}$-didehydrocytidine-5'-triphosphate tris(triethylammonium) salt (18) - ${ }^{13} \mathrm{C}\left\{{ }^{1}{ }^{H}\right\}$ NMR $\left(126 \mathrm{MHz}, \mathrm{D}_{2} \mathrm{O}\right)$

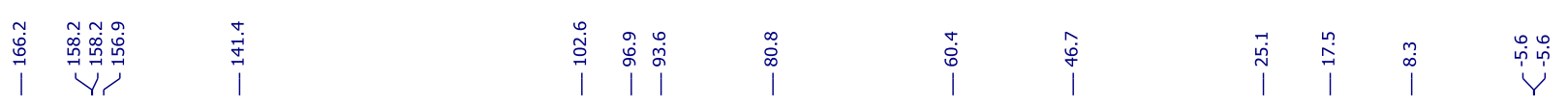

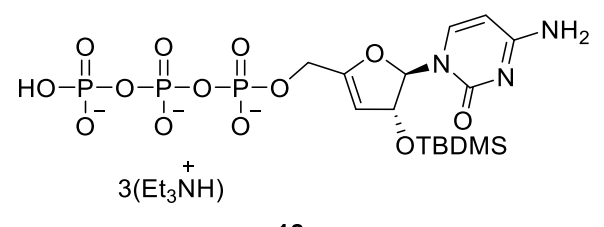

$\begin{array}{lllllllllll}200 & 190 & 180 & 170 & 160 & 150 & 140 & 130 & 120 & 110 & 100 \\ \text { f1 (ppm) }\end{array}$ 
$2^{\prime}$-O-(tert-Butyldimethylsilyl)-3'-deoxy-3',4'-didehydrocytidine-5'-triphosphate tris(triethylammonium) salt $(18)-{ }^{31} \mathbf{P}\left\{{ }^{1} \mathbf{H}\right\}$ NMR $\left(202\right.$ MHz, $\left.\mathrm{D}_{2} \mathrm{O}\right)$ نे

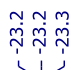

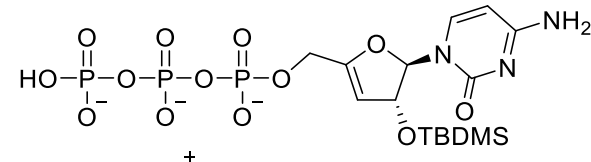

$3\left(\mathrm{Et}_{3} \stackrel{+}{\mathrm{N}} \mathrm{H}\right)$

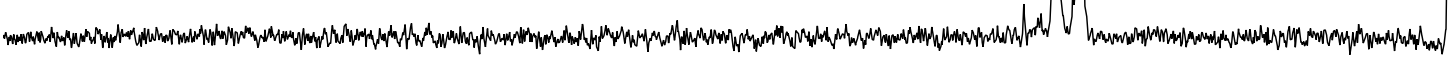

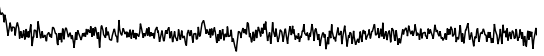

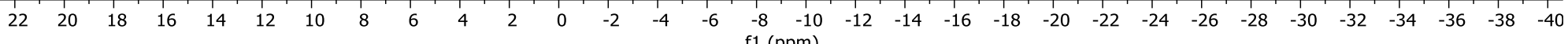


3'-Deoxy-3',4'-didehydrocytidine-5'-triphosphate tris(triethylammonium) salt (ddhCTP, 2) - ${ }^{1}$ H NMR (500 MHz, D2O)

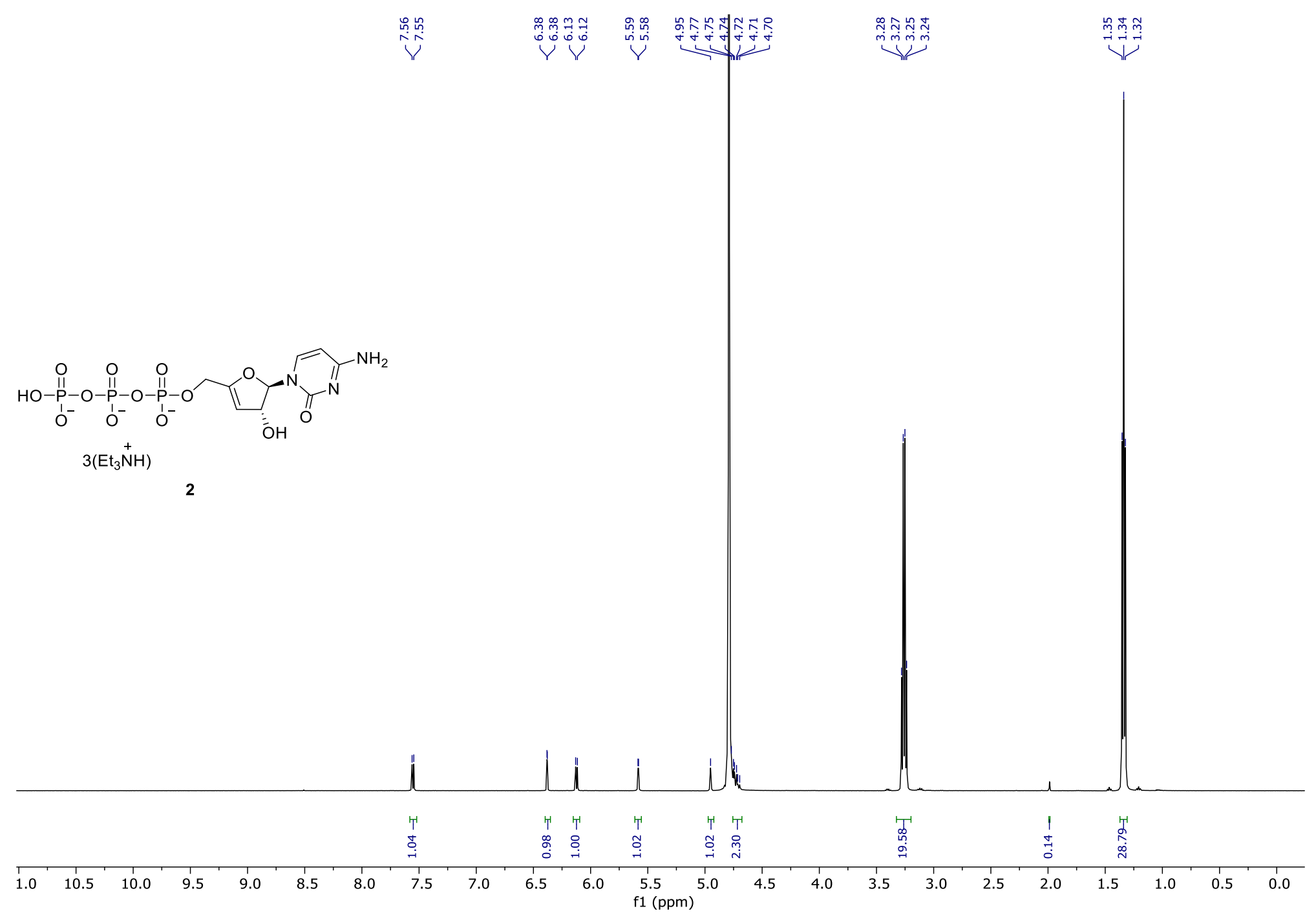


$3^{\prime}$-Deoxy-3', $4^{\prime}$-didehydrocytidine-5'-triphosphate tris(triethylammonium) salt (ddhCTP, 2) - ${ }^{13} \mathrm{C}\left\{{ }^{1} \mathrm{H}\right\} \mathrm{NMR}\left(126 \mathrm{MHz}, \mathrm{D}_{2} \mathrm{O}\right)$

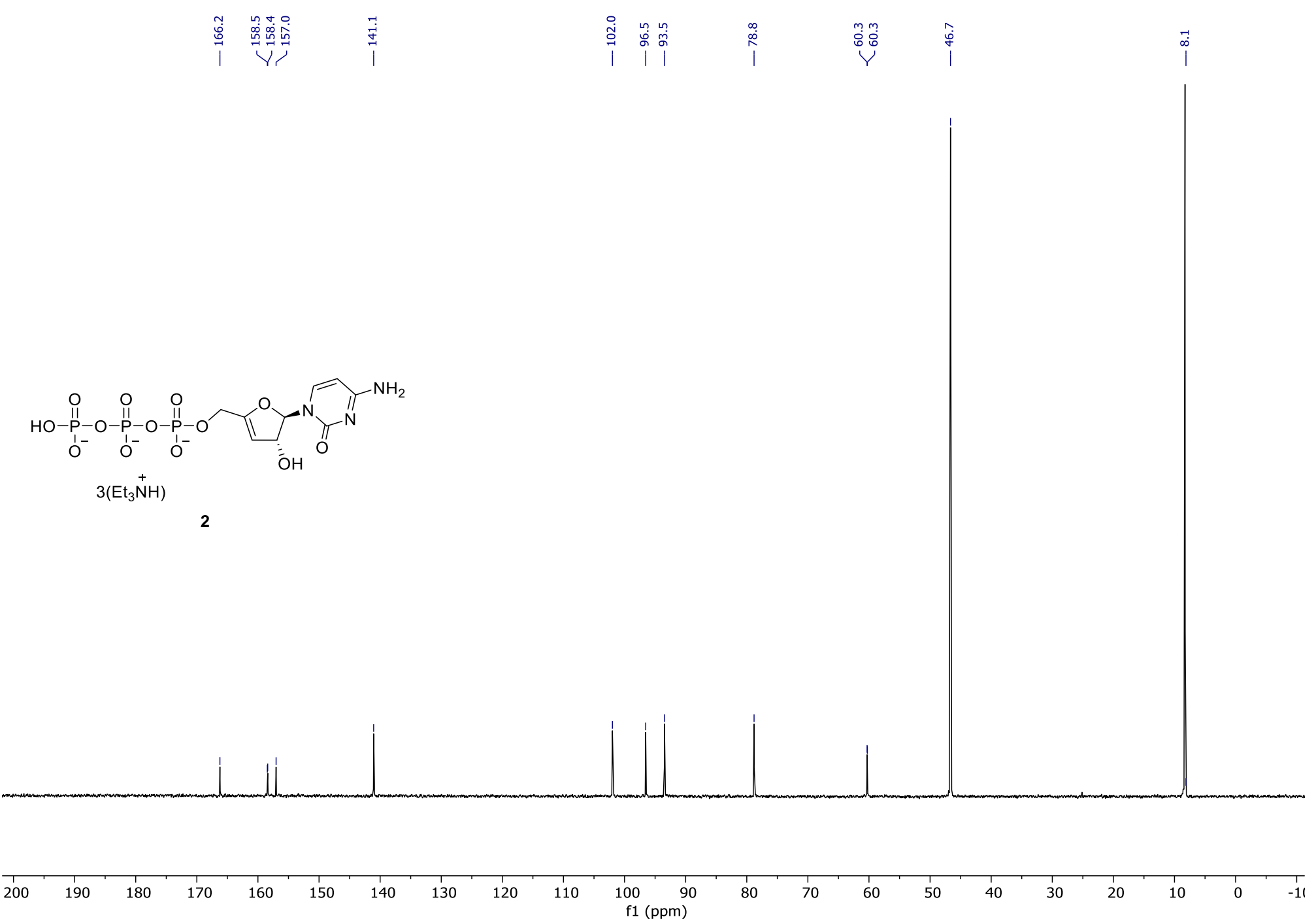


$3^{\prime}$-Deoxy-3', $4^{\prime}$-didehydrocytidine-5' -triphosphate tris(triethylammonium) salt (ddhCTP, 2) - ${ }^{31} \mathrm{P}\left\{{ }^{1} \mathrm{H}\right\} \mathrm{NMR}\left(202 \mathrm{MHz}, \mathrm{D}_{2} \mathrm{O}\right)$

$$
\text { نें }
$$

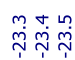
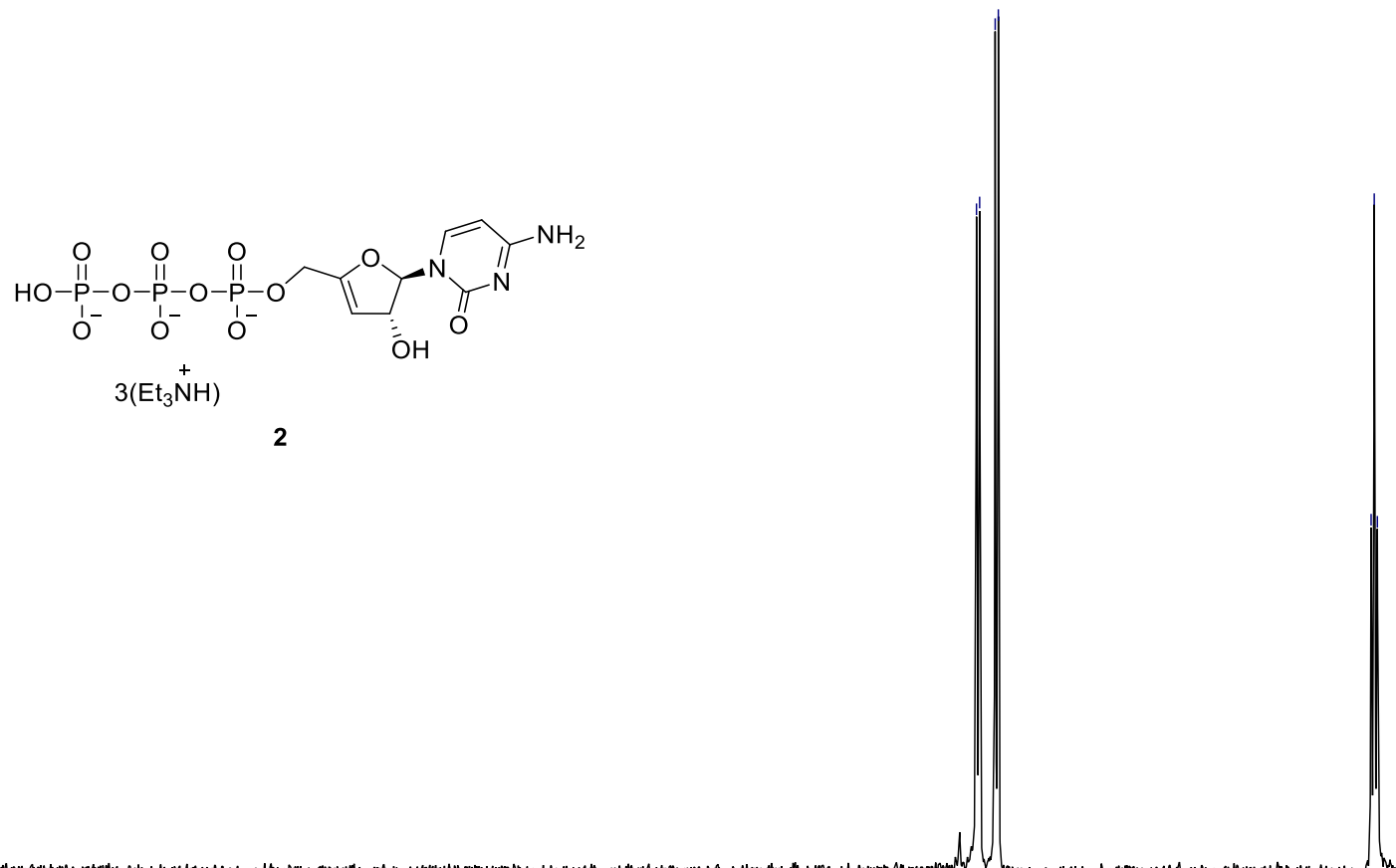

$\begin{array}{lllllll}22 & 20 & 18 & 16 & 14 & 12 & 10\end{array}$

$\begin{array}{lr}-8 & -10 \\ \mathrm{f} 1(\mathrm{ppm})\end{array}$ 
${ }^{1} \mathrm{H}$ qNMR (500 MHz, $\mathrm{D}_{2} \mathrm{O}$ ) of compound 2, with DMSO internal standard

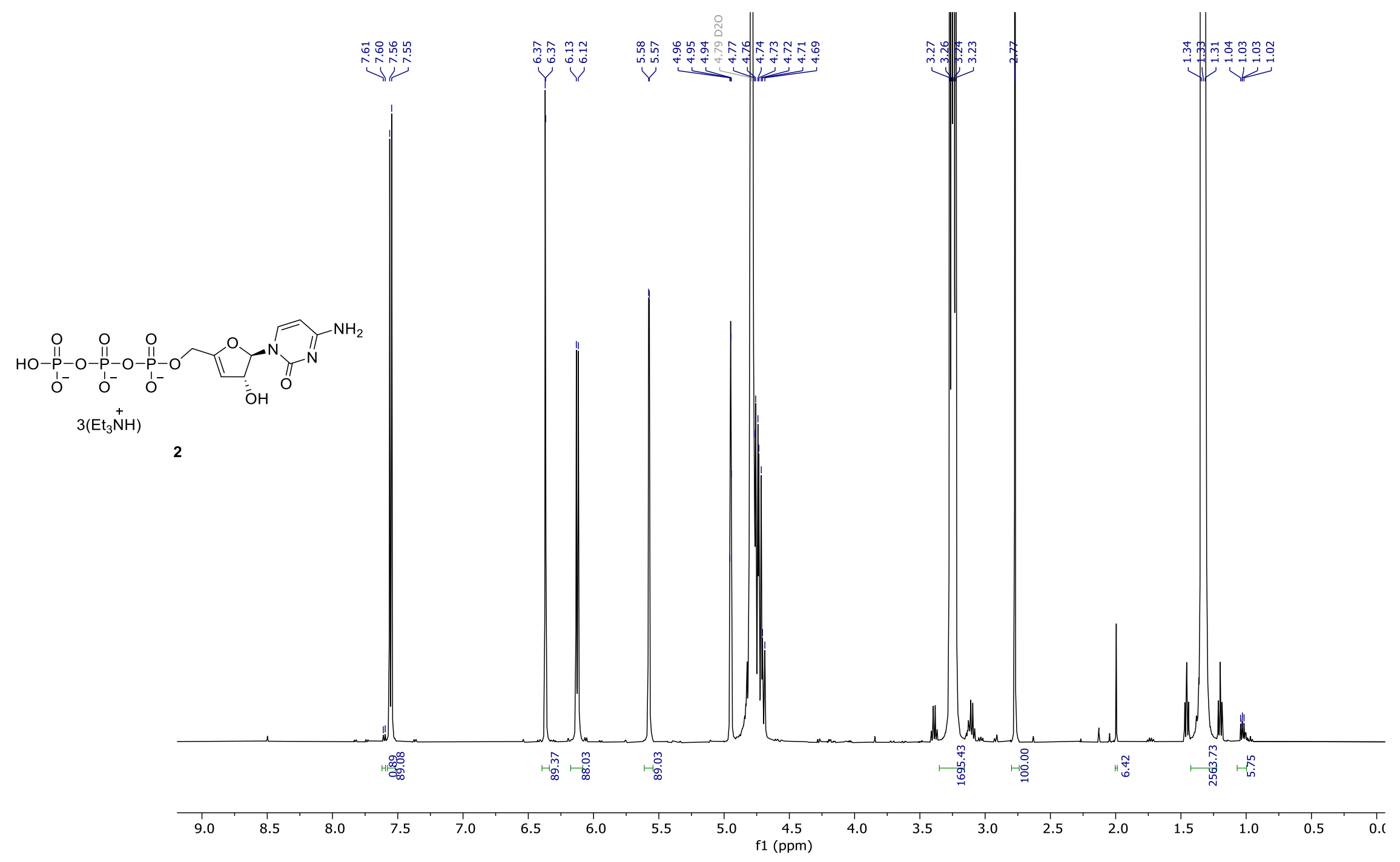

